\title{
DINÁMICAS PRODUCCIÓN-CONSUMO EN CONJUNTOS LÍTICOS: EL ANÁLISIS DE LOS CONJUNTO LÍTICOS DE LA SOCIEDAD YÁMANA
}

IVAN BRIZ

\section{RESUMEN}

En este trabajo discutimos un nuevo método para analizar los conjuntos líticos a fin de revelar las relaciones forma-función e interpretar su dimensión económica. Las tipologías tradicionales basadas en analogías etnográficas han resultado inapropiadas para comprender la variabilidad de los instrumentos líticos y fracasaron en el reconocimiento de asociaciones significativas entre la morfología de un instrumento y su uso.

En consecuencia, proponemos un método dinámico y abierto basado sobre los datos del análisis funcional, para establecer correlaciones sólidas con las variables morfotécnicas. Esta propuesta se aplica al conjunto lítico del sitio Túnel VII localizado en la costa norte del canal Beagle (Tierra del Fuego, Argentina) datado en el siglo XIX.

PALABRAS CLAVES: líticos, forma-función, dinámica, economía, yámana.

\section{PRODUCTION-CONSUMPTION DYNAMICS IN LITHICS: THE ANALYSIS OF YAMANA LITHIC ASSEMBLAGES}

\section{ABSTRACT}

In this paper we discuss a new method to analyze stone tool assemblages in an attempt to unveil form-function relationships and then interpret their socio-economic dimension. Traditional typologies which rely on assumed functions based on ethnographic analogies in order to define "types", are inappropriate to understand the variability of lithic instruments and failed in the recognition of accurately associations between tool morphology and use.

Consequently we propose a dynamic and opened method that lies in use-wear data to establish strong correlations with morpho-technical variables. This proposal has been applied to lithic assemblage of Tunel VII a shell midden located on the northern coast of the Beagle Channel (Tierra del Fuego, Argentina) and dated in XIXth. Century.

KEYWORDS: lithics, form-function, dynamics, economy, yámana.

Investigador ICREA at Dept. d'Arqueologia i Antropologia. Institució Milà i Fontanals-Consejo Superior de Investigaciones Científicas. Grupo de Investigación de Calidad AGREL de la Generalitat de Catalunya. C/ de les Egipcíaques, 15.08001. Barcelona. España.

Associated Researcher of Dept. of Archaeology. University of York. The King's Manor. York. Y01 7EP. United Kingdom. E-mail: ibriz@imf.csic.es 
El objetivo de estas páginas es exponer una propuesta metodológica de análisis de los conjuntos líticos tallados, así como mostrar los primeros resultados obtenidos en su aplicación a los materiales del sitio arqueológico Túnel VII, localizado sobre la costa norte del canal Beagle, Tierra del Fuego (Argentina), e identificado como representativo del momento de contacto entre la sociedad Yámana y las poblaciones europeas. Partimos de la premisa que los instrumentos líticos constituyen indicios materiales de las dinámicas económicas de producción y consumo desarrolladas por los grupos cazadores-recolectores-pescadores de la región. Consecuentemente, es necesario ajustar los procedimientos analíticos utilizados por la arqueología de tal forma que esa dinámica sea puesta en evidencia. Desde nuestra perspectiva, es a partir de las relaciones forma-función que podemos acercarnos a la organización de esa dinámica producción-consumo y, a partir de ahí, a las prácticas sociales concretas que se desarrollaron en el sitio.

Si nuestro objetivo de análisis último como disciplina científica, es el reconocimiento de las formas de organización socio-económica del pasado, necesitamos articular un aparato clasificatorio y analítico capaz de poner en evidencia la materialidad arqueológica en clave de esas modalidades de organización social. Modalidades que son entidades dinámicas en base a los procesos históricos de los cuales son, al mismo tiempo, sujeto y objeto. En el caso del estudio de los conjuntos instrumentales líticos y sus procesos de producción, para alcanzar esos objetivos de interpretación en clave social, necesitamos que su análisis y clasificación se desarrollen también en función de los mismos.

Es a partir de nuestro marco teórico substantivo (Marx 1992), que consideramos que el primer paso para el análisis de las dinámicas sociales de un grupo humano debe concentrarse en la economía del mismo. No significa este hecho que no se considere relavante el estudio de los mecanismos de reproducción social, sino que, focalizándonos inicialmente en el ciclo económico, conseguiremos establecer las bases de supervivencia material sobre las que se asienta el grupo, generando el resto de elementos y rasgos sociales que lo constituyen. Sin olvidar que la relación entre ambas esferas es constante y mútuamente definidora. En base a estas premisas, en nuestro análisis de los conjuntos líticos perseguimos el reconocimiento de las dinámicas entre la esfera de la producción y la del consumo. Obviamente, el conjunto de instrumentos líticos tan sólo será uno de los diversos elementos que participan, constituyéndola, en la actividad económica humana.

Con la finalidad de intentar ofrecer una visión clara de la propuesta, nos focalizaremos en presentar los procedimientos generales del método de análisis utilizado, en especial sobre tres elementos: a) su articulación lógica; b) la metodología de análisis morfológico y morfotécnico de las piezas, así como sus posibilidades de identificación y representación de la variabilidad; $y$, finalmente, y en menor grado, c) el instrumento matemático empleado para la identificación de las dinámicas forma-función. Por ello, nos concentraremos única y exclusivamente en estas páginas en el análisis de los filos que presentan evidencias de uso ${ }^{1}$. De la misma manera, y dadas las características formales de estas páginas, no explicitaremos (ni emplearemos sus resultados) ni el estudio de las materias primas empleadas, ni las volumetrías de los instrumentos. En relación al estudio de las materias primeras, remitir al importante trabajo desarrollado por X. Terradas (1996), mientras que en relación a las volumetrías, los resultados ya han sido expuestos en anteriores trabajos (Briz 2004).

\section{TÚNEL VII; UNA EXCAVACIÓN (ETNO)ARQUEOLÓGICA}

El sitio Túnel VII fue excavado a lo largo de diversas campañas arqueológicas en el marco de dos proyectos etnoarqueológicos hispano-argentinos (Vila 2004), bajo la co-dirección de Jordi Estévez (Universitat Autònoma de Barcelona), Luis Abel Orquera (Asociación de Investigaciones AntropológicasCONICET), Ernesto Luis Piana (Centro Austral de Investigaciones Científicas-CONICET) y Assumpció Vila (Institució Milà i Fontanals-CSIC). Nuestra propuesta de Etnoarqueología (Vila y Piana 1993; Estévez y Vila 1996; Vila y Estévez 2001; Vila 2004; Briz 2010) se articula mediante la contrastación entre fuentes etnográficas e información arqueológica sobre un mismo caso de estudio. El objetivo de la confrontación entre ambas esferas de conocimiento

1 En la propuesta general podemos revisar la articulación del análisis global de las piezas, incluyendo también el resto de los filos (Briz 2004 y 2007). 


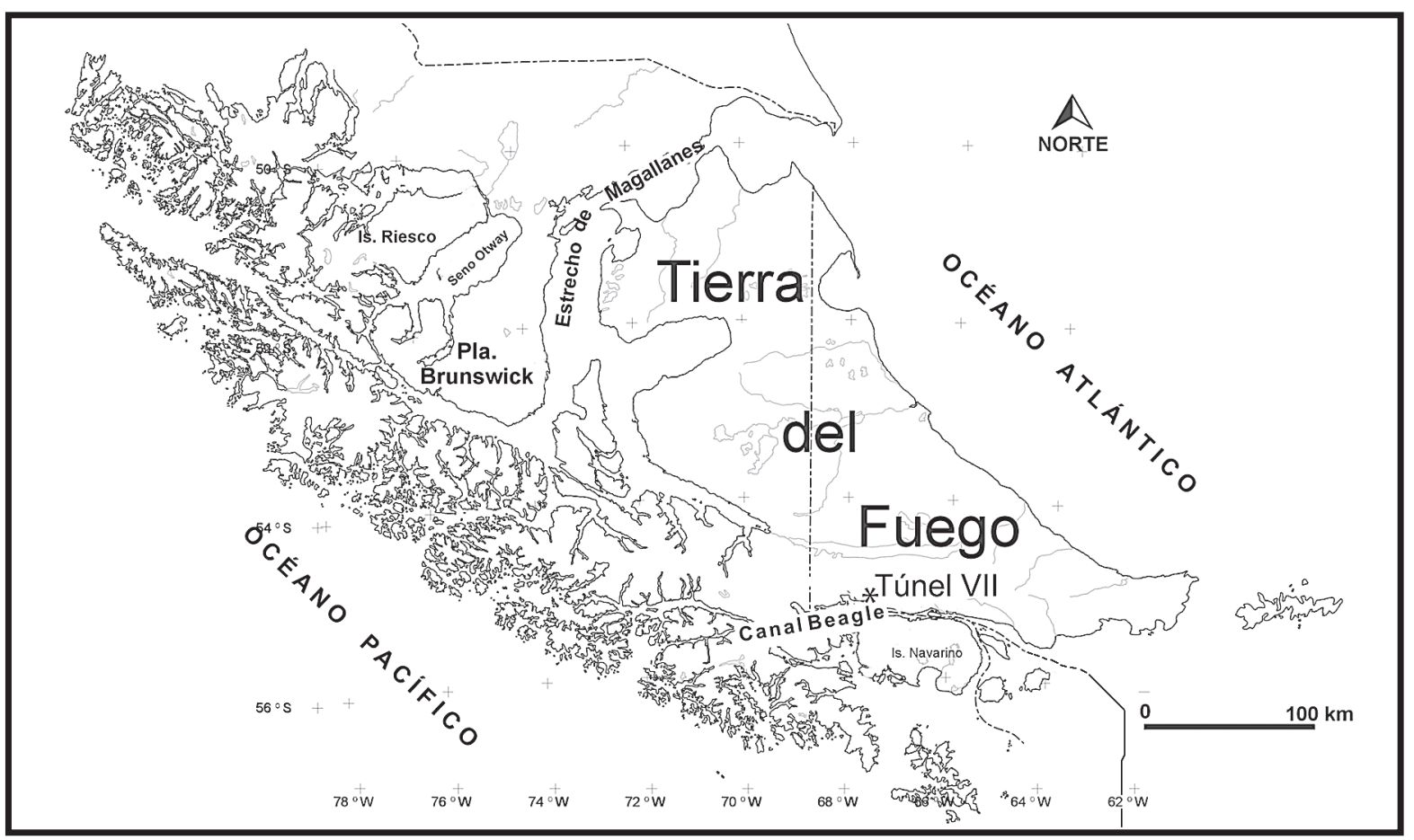

Fig. 1. Ubicación de Túnel VII en Tierra del Fuego.

(ethnos versus arjeos) es disponer de una lectura crítica de la información aportada por etnografía, etnología o antropología, así como obtener desarrollos metodológicos para incrementar cualitativa y cuantitativamente nuestras capacidades interpretativas en arqueología que incluyen la mejora cualitativa en la generación de hipótesis investigadorasasí como de nuevas metodologías (ver entre otros: Terradas 1996; Zurro 1996 y 2010; Clemente 1997; Piqué 1997; Vila y Estévez 2001). Este incremento de nuestra capacidad explicativa tiene dos propósitos: retornar a los contextos de la prehistoria del Viejo Mundo para mejorar nuestra capacidad de explicación (Briz et al. 2009) y permitirnos conocer mejor las formas de vida de las sociedades cazadoras-recolectoras de la región del canal Beagle.

Túnel VII (54 49'15"S-68 09' 20”W) se encuentra ubicado en el interior de la estancia Túnel, en la costa norte del Canal Beagle, en el actual territorio de la provincia de Tierra del Fuego (Argentina) (Orquera y Piana 1996: 47). Se trata de un un conchero antropogénico representativo del paisaje arqueológico de la región de los canales fueguinos (Orquera y Piana 1999) y de los patrones micro-espaciales de asentamiento de la sociedad
Yámana (Estévez y Vila 2000). La complejidad de una puesta en común de los métodos de trabajo de dos equipos de investigación provenientes de ámbitos y tradiciones diferentes generó un interesante debate metodológico en cuanto a la producción del registro arqueológico; en especial, en relación a la interpretación estratigráfica (Orquera y Piana 1996a; Vila et al. 1996a; Estévez y Vila 1998 y 2000). Para estas páginas, consideraremos Túnel VII como un sitio conformado por una sucesión de diez episodios de ocupación diferenciados entre sí, y con ausencia de actividades antrópicas materialmente reconocibles entre sí (Estévez y Vila 2006). Los episodios (o momentos) de ocupación son reconocidos y vertebrados en la presencia de un número, como mínimo, idéntico de áreas de combustión en la depresión interior de la estructura anular, hipotéticamente definida como área de hábitat (Orquera 1996; Estévez y Vila 2000: 51-52)2 . Otro indicador de la existencia de estos diez momentos de ocupación diferenciados es la depositación de sedimento no antropogénico

2 Los episodios han sido denominados en una secuencia alfabética a partir de la letra A, episodio inicial de ocupación del sitio (Estévez y Vila 2006). 
entre unidades estratigráficas, o bien alteraciones en las depositaciones de matriz antropogénica en la formación del conchero que permiten identificar momentos diacrónicos (Orquera y Piana 1999 y 2000). La caracterización de estos episodios, sin embargo, es coherente con la complejidad estratigráfica de este tipo de sitios arqueológicos: los límites estratigráficos de las diferentes ocupaciones no siempre se han podido delimitar con claridad, por lo cual algunas unidades estratigráficas no pudieron ser asignadas unívocamente a un momento de ocupación. La cronología del sitio 100_45AP (AC 871) (Orquera y Piana 1999: 35-36) lo ubica en una franja temporal de contacto y ocupación de Tierra del Fuego por el mundo industrial (Orquera 1995: 108-109; Estévez y Vila 2000: 46).

\section{UNA PROPUESTA METODOLÓGICA}

Necesitamos reconocer la naturaleza dialéctica, de la materialidad arqueológica, en tanto que forma parte de una realidad cuya más destacada característica es su dinámica (De Gortari 1965 y 1970). En el caso de los conjuntos líticos tallados, intentamos dejar a trás el uso del acto clasificatorio morfológico, o supuestamente morfo-funcional, como elemento básico de análisis (Adams 1988; Andrefsky 1998; Pie y Vila 1992; Merino 1994) para intentar aprehender las relaciones sociales de producción y consumo contenidas en cada instrumento lítico (Briz 2002). Todo elemento constituyente de la materialidad arqueológica es, en última instancia, la materialización de esas relaciones sociales de producción y consumo, que son la base inicial para la construcción de toda relación social en cualquiera de sus manifestaciones.

$\mathrm{Al}$ asumir la naturaleza económica de los conjuntos líticos (Risch 2002) podremos construir las metodologías adecuadas para mostrar adecuadamente la naturaleza dinámica del registro. Esta dinámica económica es correlato de la dinámica social en tanto que es producto y materialización del trabajo humano socialmente articulado (Pfaffemberger 1992). Lo social no se restringe únicamente a lo económico, pero lo económico está presente en todos y cada uno de los ámbitos, manifestaciones o materialidades de lo social.

Todo producto sobre soporte mineral es producido para incorporarse al proceso productivo general
(Marx 1992) como medio de producción (es decir, bien como materia auxiliar, bien como instrumento de producción (Briz et al. 2002)). El objetivo final de los procesos de talla, como procesos de producción que son, es la producción de instrumentos de trabajo para ser incorporados a nuevos procesos de producción (Lumbreras 1984a y b).

En todo instrumento lítico podemos reconocer su doble naturaleza: es producto final de un proceso de producción, y también instrumento de trabajo que se incorpora, al ser empleado en un proceso de trabajo, en el producto resultante (Clemente et al. 1996; Briz 2002). Así, en una misma unidad material, tenemos identificado el ámbito de interrelación de estas dos esferas: producción y consumo se manifiestan en el instrumento, como elemento que es en la dinámica económica y que al mismo tiempo la constituye (Marx 1992). Esto es: el trabajo humano bajo condiciones socio-históricas específicas.

La morfología de un artefacto es el producto final del proceso de trabajo, tecnológicamente articulado (Ingold 1997 y 2001). La función es la materialidad resultante de la dinámica del proceso de consumo en el que ese artefacto fue empleado, convirtiéndose en instrumento ${ }^{3}$ (Semenov 1981). Los rastros de uso macro y microscópicos, objeto del análisis funcional (Semenov 1971 y 1981; Korobkova 1983; Vila 1985; Mansur 1987; Vila y Clemente 2000; Álvarez 2003, Mansur y Lasa 2005; entre muchos otros), son la evidencia material de esta interactuación entre ambos ámbitos: en los rastros de uso encontramos tanto el indicio de la materia trabajada y las condiciones en las que fue trabajada (dureza de la materia, tipo de materia), como los indicios del trabajo generado por los seres humanos y multiplicado por el instrumento de producción (cinemática del trabajo desarrollado): son la evidencia material del trabajo. Cualquiera de los rasgos del proceso de producción del artefacto (retoques, bulbo....) como del proceso de consumo del mismo (micropulidos, estrías, ...), cuyo uso establece su función, son las improntas materiales

3 A la vez que son sus opuestos: morfología del ítem que nos puede permitir reconocer el consumo de materías primas, instrumentos y trabajo para la obtención de los artefactos (desde la obtención de materia prima hasta el producto final). Función del instrumento que nos permite reconocer la producción, el proceso de trabajo, en donde ha participado al ser usado. 
del trabajo, socialmente articulado (tecnología) que se implementó (Merino 1994).

El reconocimiento y la búsqueda de relaciones forma-función en el instrumental lítico no es novedoso. Desde la aparición de las capacidades del análisis funcional de huellas macro y microscópicas de la mano de S. A. Semenov, diversos esfuerzos se concentraron en superar las interpretaciones de base morfo-funcional construidas en base a la analogía etnográfica, o el paralelo morfológico. La obtención de marcadores independientes de uso permitía una mayor aproximación a la "vida económica" de los conjuntos líticos. Intentar delimitar pautas de vinculación entre morfologías productivas y los usos en los que habían sido empleadas se mostraba como un destacado elemento de evaluación de la efectividad tecnológica de los casos a estudiar. Así, desde diferentes perspectivas teóricas, las pasadas décadas de los ochenta y noventa vieron un intenso esfuerzo en la determinación de las relaciones forma-función (Odell 1981; Juel Jensen 1988; Knecht 1988; Finlayson y Mithen 1997; Calvo 1999 y 2002; Landini et al. 2000; Carbonell y Rodríguez 2002; Mansur y Lasa 2005 ; entre otros). A partir de los primeros años del presente siglo, los esfuerzos de investigación de esta línea fueron paulatinamente decayendo, sobre todo por la ausencia de resultados evidentes.

Dos son los elementos que, a nuestro criterio, impidieron a aquellas líneas de trabajo alcanzar sus objetivos. En primer lugar, el uso de procedimientos de clasificación inadecuados para los objetivos que se habían planteado. El mantenimiento de las categorías tipológicas como unidades básicas de análisis, supeditaba y forzaba la identificación de posibles vinculaciones entre forma y función. Uno de los ejemplos que mejor muestran esta problemática es el trabajo de H. Knecht (1988) sobre los buriles: asumir el tipo "buril" como unidad básica de definición sesgaba el análisis. Al enfrentar los datos proporcionados por el análisis funcional con la caracterización morfológico-tipológica, los resultados apuntaban a una elevada diversidad de posibles usos, sin identificar una posible correlación forma-función. Trabajos posteriores (Calvo 1999 y 2002; Landini et al. 2000; Debert y Sherriff 2007; Hardy et al. 2008; Pawlik 2009) han tenido que enfrentarse al mismo problema en mayor o menor grado, desde diferentes posicionamientos teóricos, y con mayor o menor capacidad de resolución.
El segundo de los elementos clave que limitó los resultados fue el desarrollo de planteamientos carentes de una visión ajustada y necesaria de la complejidad del problema a tratar. Gran parte de los trabajos citados habían previsto resultados unívocos, de baja variabilidad. Es decir: intentaban identificar correlaciones directas entre forma y función del instrumental lítico, sin tener en cuenta que podía existir una variabilidad en la intensidad de esa correlación. Asumir la necesidad de reconocer diferentes tipos de especialización morfo-funcional era un elemento básico para poder aproximarse a una realidad arqueológica (Briz 2004): la existencia de pautas forma-función podía ser identificable a partir de las dinámicas de esa correlación y no a partir de estados de esa misma correlación. Es decir, mediante la utilización de tipologías cerradas se redujo la posibilidad de hallar asociaciones significativas entre formas y usos. Y, al mismo tiempo, para este cambio de perspectiva se hacía necesario, nuevamente, organizar el material y la investigación desde una posicionamiento fijado en la dinámica y el cambio (De Gortari 1970; Bate 1982), en vez de en situaciones estáticas unívocas.

Es a partir de estas premisas previas que consideramos factible articular una metodología cuyo objetivo es la identificación, en cada instrumento lítico tallado, de su naturaleza dinámica y económica. La unidad de análisis propuesta es el filo activo de cada instrumento mientras que la unidad de interpretación es el conjunto lítico completo representativo de un deteminado momento de ocupación (Briz 2004). En trabajos posteriores, este análisis nos permitirá caracterizar las formas de organización del trabajo a nivel regional y comparar su dinámica a través del tiempo.

Tres son los campos en los que necesitamos trabajar para esta implementación metodológica: 1) la identificación de los procesos de trabajo desarrollados por el instrumental lítico; 2) la caracterización analítica de las morfologías de los instrumentos, mediante una sistemática metodológica cuantificable o cualificable, unívoca y revisable; y 3) el desarrollo de una metodología estadística capaz de reconocer y asumir la dialéctica y la variabilidad interna del conjunto a analizar. El primer ámbito es el del análisis funcional. Pero implementado en base a su planteamiento metodológico original (Semenov 1971 y 1981; Longo 
y Skakun 2005) y no en su articulación reductora a la simple práctica técnica (como ya pusieron de relieve Vila y Clemente (2000)).

Tenemos que remarcar que, en última instancia, la propuesta de Semenov no persigue la identificación de las materias trabajadas por el instrumental lítico, sino la identificación y análisis del trabajo humano en base a sus restos materiales (Semenov Op. Cit.; Korobkova 1983). No revisaremos aquí los fundamentos del análisis funcional de base microscópica. En primer lugar, porque se trata de una línea de investigación consolidada nada novedosa para los contextos arqueológicos que trataremos, dándose, además, que la Isla Grande de Tierra del Fuego debe ser uno de los puntos de Sudamérica con mayor presencia de esfuerzos y resultados en análisis funcional. En segundo lugar porque páginas mucho mejores que estas han explicado adecuadamente las bases de este tipo de análisis (Semenov 1981; Korobkova 1983; Mansur 1987; Vila 1987; Álvarez 2003).

El segundo ámbito de trabajo es el desarrollo de una metodología adecuada para el análisis de las morfologías. Estos análisis morfológicos nos ofrecen resultados analíticos morfotécnicos de un producto que es resultado de un proceso de producción tecnológica (Briz 2007; Hiscock 2007). Es desde esta perspectiva que proponemos una sistemática de trabajo cuantificable/cualificable y unívoca, analítica, que nos permita reconocer las morfologías existentes como productos del trabajo humano, es decir, que ponga de relieve los rasgos morfológicos que son relevantes en la vida económica del instrumento tanto como producto como instrumento de trabajo. Los trabajos de Georges Laplace (1956, 1964, 1966 y 1972) para el desarrollo de la Tipología Analítica, basada en la dialéctica como lógica procedimental, resultan la vía analítica adecuada para enfrentar la problemática señalada en estas páginas (Vila 1977 y 1986; Pié y Vila 1992; Vila et al., 1996a). La propuesta laplaciana reconoce, al mismo tiempo, las interrelaciones y jerarquías existentes entre los diferentes rasgos que conforman cada pieza (Laplace 1972 y 1974). Es a partir de la identificación de los rasgos indicativos del proceso de producción del artefacto (morfología (incluyendo la materia prima) y morfometría) que se genera una identificación jerarquizada y sintáctica de la pieza (Briz 2004: 283); permitiendo, una vez analizado el conjunto, su caracterización en base a sus dinámicas internas (Laplace 1964, 1972, 1974, 1975 y 1981; Sáenz de Buruaga, 1991).

Dos elementos esenciales a destacar de la lógica del método: en primer lugar la escala de análisis es una decisión que ha de ser coherente en base a los objetivos, explicitados, de la investigación: ésta es la dialéctica entre sujeto investigador y objeto. En segundo lugar, el método laplaciano es abierto: susceptible de ser modificado, ampliado, corregido, reajustado, para ser aplicado a diferentes contextos y respondiendo a preguntas diferentes (Vila, 1977 y 1986). En nuestro caso, este método se desarrolla articuladamente con el análisis funcional (Vila 1977) e incorpora al análisis la totalidad de los ítems que presentan rasgos de haber sido producto de un proceso de talla sin limitarse a las piezas retocadas y núcleos. El análisis funcional ya ha demostrado de forma más que efectiva la invalidez de la asociación directa entre la presencia de retoque y la naturaleza instrumental de una pieza: piezas sin formatización secundaria han sido efectivamente empleadas; así mismo, tampoco es correcta una correlación directa sin contrastación entre morfologías y usos (Vila 1987; Vila et al. 1996: 273).

\section{EL ANÁLISIS MORFOTÉCNICO}

Determinada la materia prima sobre la que se ha realizado una pieza, necesitamos analizar los rasgos morfológicos y morfotécnicos que la caracterizan. La propuesta nos permite descomponer los rasgos constituyentes de todo ítem en diferentes variables cualitativas o cuantitativas contrastables. Los rangos de análisis se establecen en dos niveles: obtención de la pieza y morfología de la pieza. En el primero, se identifica la materia prima y se establece un reconocimiento en base a la obtención del soporte (asimiliable a los tipos primarios de Laplace: Sáenz de Buruaga 1991: 41): lasca, fragmento, núcleo o nódulo. En el caso de las dos primeras categorías, pueden ser diferenciadas en un segundo nivel de análisis mediante: a) la identificación de la presencia de formatización secundaria que indica los niveles de inversión de trabajo realizada; b) la inclusión de una valoración tipométrica de primera aproximación: lascas y fragmentos laminares; lascas y fragmentos secundarios de talla (cuya medida mayor sea menor a $20 \mathrm{~mm})$. 
Ahora bien, necesitamos articular un reconocimiento de la variabilidad morfológica interna de cada pieza y sus interrelaciones. La dinámica de análisis segmenta la pieza en sus cuatro filos y se evalúan los rasgos que conforman cada filo y sus interrelaciones. Así el resultado para una lasca retocada sobre cinerita, a nivel de la formulación, sería (orientada en base al talón o filo proximal y empleando el eje de percusión como eje longitudinal (Laplace 1976 y 1977):

LR [filo izquierdo+filo distal+filo derecho] Tipo de talón. Materia prima. Volumetría.

Si la pieza analizada fuese un fragmento $y$, consecuentemente, no poseyera talón, la formulación sería:

LR [filo izquierdo+filo distal+filo derecho+filo proximal] Materia prima. Volumetría.

¿Cuáles son las variables propuestas para el análisis? Tipo de filo, modo (ángulo del filo), delineación del filo y orientación del filo (Vila 1986). La primera de las variables, propuesta por A. Vila (Op. cit.), responde a la necesidad de incorporar al análisis aquellos elementos que, carentes de formatización secundaria, no habían sido objeto de interés para la propuesta original (Laplace 1972). Ahora bien, la naturaleza abierta del método no impide que estas variables, válidas para el análisis del conjunto de Túnel VII, puedan ser ampliadas con otras categorías en otros conjuntos líticos. En la tabla 1 se resumen los valores de cada variable.

La articulación del análisis, y su representación, se desarrolla avanzando por los filos (y en cada filo) desde el segmento proximal del filo izquierdo y avanzando según el sentido de las agujas del reloj, en el siguiente orden: tipo de filo, modo, delineación orientación.

Veamos un ejemplo de la formulación. Un filo constituido por un ángulo de $46^{\circ}$, rectilíneo, convergente sería representado:

[aArect cvg]
Delimitadas las variables, la sintaxis de análisis (Laplace, 1972) es el instrumento que permite expresar la complejidad y variabilidad de un filo y una pieza. Un ejemplo puede sernos de gran ayuda: tomemos el filo abrupto rectilíneo convergente antes mencionado. Supongamos que, en realidad, el filo posee una mayor complejidad: avanzando por ese filo izquierdo desde el segmento proximal hasta el distal, encontramos un retoque plano, marginal, directo, sobre una concavidad convergente. La formulación sería la siguiente:

[aArect cvg-rPmdcc cvg]

donde el símbolo "-“" nos indica la continuidad y sucesión entre una morfología y otra. La información puede (debe) ser simplificada para una mayor operatividad, eliminando aquellas informaciones recurrentes. En nuestro ejemplo la orientación de ambos segmentos diferentes del filo es la misma: podemos eliminar las recurrencias entendiendo que una determinada variable es representativa de los segmentos previos. Así nuestra fórmula sería:

[aArect-rPmdcc cvg].

Si nuestro filo continúa con una fractura simple sinuosa paralela y, después, convergente. El resultado sería:

[aArect-rPmdcc cvg-fSsin par-cvg].

La totalidad de nuestra pieza hipotética podría ser un filo distal en un ángulo cero (terminado en punta), mientras que el filo derecho es un retoque plano muy marginal inverso rectilino paralelo. La pieza, sobre cinerita, posee un talón facetado. Sus medidas son: longitud: $23 \mathrm{~mm}$, anchura: $16 \mathrm{~mm}$ y un grosor de $6 \mathrm{~mm}$. referidas a las medidas máximas del eje longitudinal, el ancho y el grueso (Vila 1986). Su representación global es:

LR[aArect cvg-rPmdcc cvg-fSsin parcvg+.+rPmmirect par] TFA 23/16/6 CIN.

En cada pieza se considera los rasgos de extracción por talla, el conjunto de formulas analíticas de sus filos, y la volumetría, manteniendo la concepción

Tabla 1. Variables del análisis morfotécnico.

\begin{tabular}{|c|c|c|c|c|c|}
\hline Tipo de filo & Modo & $\begin{array}{l}\text { Amplitud del retoque } \\
\text { (para piezas } \\
\text { retocadas) }\end{array}$ & $\begin{array}{c}\text { Dirección del retoque } \\
\text { (para piezas } \\
\text { retocadas) }\end{array}$ & Delineación & Orientación \\
\hline $\begin{array}{c}\text {-ángulo (a), } \\
\text {-retoque (r), } \\
\text {-fractura (f), } \\
\text {-charnela (cha) } \\
\text {-ángulo cero o } \\
\text { punta (.) }\end{array}$ & $\begin{array}{c}\text { 30 } \text { Planos }(\mathrm{P}), \\
\text { 31-45응 Simples (S) } \\
>45^{\circ} \text { Abrutos }(\mathrm{A})\end{array}$ & $\begin{array}{l}\text { muy marginal }(\mathrm{mm}) \\
\text { marginal }(\mathrm{m}) \\
\text { profundo }(\mathrm{p}) \\
\text { muy profundo }(\mathrm{pp})\end{array}$ & $\begin{array}{l}\text { directo (dir), } \\
\text { inverso (i), } \\
\text { bifacial (bi) } \\
\text { alterno (alt) }\end{array}$ & $\begin{array}{l}\text { rectilínea (rect), } \\
\text { convexa (cx), } \\
\text { cóncava (cc) } \\
\text { sinuosa (sin) }\end{array}$ & $\begin{array}{c}\text { paralelo (par), } \\
\text { divergente (div), } \\
\text { convergente (cvg) } \\
\text { transversal (trans) }\end{array}$ \\
\hline
\end{tabular}


de análisis global de la propuesta inicial. Se obtiene así la descomposición relacional de los rasgos de forma jerarquizada y sistemática (pero al mismo tiempo ajustable a nuestra necesidad investigadora). Existen más elementos de incremento y mejora de la información sintáctica, en tanto que correlato de la variabilidad de cada pieza. Para mayor detalle se puede profundizar en trabajos anteriores (Laplace 1964, 1972 y 1974; Vila 1977, 1981 y 1986; Vila et al. 1996; Briz 2004 y 2007).

\section{FORMA Y FUNCIÓN}

Dos son las cuestiones básicas a tener en cuenta en el momento de articular cómo enlazar nuestra hipótesis con el conjunto lítico, ahora caracterizado analíticamente. En primer lugar, pretendemos buscar las pautas y regularidades de las morfologías empleadas en procesos de trabajo determinados. Es decir, en la contrastación de nuestra hipótesis, el desarrollo del proceso de trabajo posee rango de variable independiente (Briz 2004). Organizaremos nuestra búsqueda de pautas en base a los procesos de trabajo desarrollados y la caracterización analítica de éstos: cinemática de trabajo, dureza de la materia trabajada y en aquellos casos factibles, material trabajado (Mansur 1987; Clemente 1997;). Este análisis se aplicará tratando por separado a los instrumentos con formatización secundaria de los carentes de ella. La mayor inversión de trabajo en el retoque para un determinado proceso de trabajo, puede ser así evaluada y medida. En segundo lugar: se asume la variabilidad interna de las relaciones forma-función. Para poder reconocerlas, emplearemos dos escalas de análisis. El primero, estrictamente aproximativo del conjunto, basado en una estadística descriptiva (porcentajes, etc...) y concentrada en el desarrollo de una heurística de las tendencias de correlación a nivel macro. El segundo, articula un reconocimiento de la estructuración interna de la variabilidad de cada una de las variables morfotécnicas, en base a su presencia en un determinado proceso de trabajo.

Para cada proceso de trabajo, compilamos los rasgos morfotécnicos de los filos de cada una de las variables, cuantificando el número de casos presentes para cada uno de los valores de cada variable. Consideraremos relevantes todas las variables morfotécnicas de los filos a excepción de la orientación, que consideraremos no relevante en tanto que puede ser modificada por el modo de aprehensión del instrumento (Briz 2002). En los casos que exista diversidad de valores de una misma variable, analizaremos el conjunto de valores del filo concreto, determinando cuál es la tendencia general existente, inclusive si el valor resultante es la ausencia de una tendencia clara, lo que denominaríamos valor mixto (Briz 2004).

Asumimos la existencia de un nivel de variabilidad en la pauta de correlación forma-funcion de cada rasgo morfológico. Si la pauta resultante nos ofrece una única correlación, esta quedará perfectamente reflejada en nuestros análisis, pero también existe la posibilidad de una diversidad de opciones tecnomorfológicas a producir/emplear. Si existe tal variabilidad, necesitamos reconocer su organización y jerarquía interna como rasgo significativo de la diversidad morfológica lítica producida. Eso permitirá reconocer la relevancia de determinados valores, desde el rasgo más típico de un determinado proceso de trabajo, hasta el más alejado de la significación específica, obteniendo una imagen de las dinámicas forma-función allí implementadas.

Es importante que nos detengamos en este punto: a diferencia de las propuestas del procesualismo británico (Clarke 1984), lo que estamos realizando no es una segmentación analítica de los componentes principales para, posteriormente, "recomponer" el tipo (Merino 1994: 277). Sobre todo, porque nuestro objetivo, a diferencia de la propuesta anglosajona, no es reconstruir el tipo mediante métodos de objetivación cuantificadora. Intentamos conseguir una caracterización analítica del conjunto en base a su relación forma-función.

El instrumento estadístico empleado para este reconocimiento de la estructuración de la variabilidad interna es la Dinámica del Análisis Estructural, (Laplace 1974, 1975, 1978, 1981; Laplace y Livache, 1978). La dinámica está conformada por el reconocimiento jerarquizado de los diferentes grupos presentes en un ámbito de medición (en este caso, la variabilidad dentro de un rasgo morfotécnico), con el objetivo final de identificar la estructura del conjunto en base a su equilibrio específico interno. La propuesta de la Dinámica del Análisis Estructural está constituida por diferentes instrumentos y estadios de análisis estadístico (Laplace, 1974; Laplace y Livache 1978; para un excelente compendio en castellano: Sáenz de Buruaga 1991: 62-76). El procedimiento de cál- 
culo organiza el reconocimiento jerarquizado de los diferentes rasgos contituyentes de cada variable por separado, analizando la significación matemática de las diferencias existentes entre estos rasgos y reconociendo cuantitativamente la importancia del mismo (en base al número de casos que lo componen) e identificando sus (inter)relaciones con los restantes rasgos (Laplace 1975 y 1981). Dos son los elementos de análisis a emplear en este punto: la secuencia estructural (Laplace 1974) y el cálculo del lien (Laplace 1980). Concentraremos nuestra atención únicamente en el primero, que es el que hemos aplicado al conjunto de Túnel VII. El cálculo, realizado mediante el empleo como instrumento básico del test y la distancia del $\mathrm{X}^{2}$ (diferencia entre distribución teórica y la real), identifica las diferencias significativas (llamadas rupturas) de un grupo respecto a los restantes del conjunto, midiendo la intensidad de esta diferencia (Laplace 1974, 1975 y 1981; Vila 1986; Sáenz de Buruaga 1991). En el caso de conjuntos con bajo número de casos, existen alternativas estadísticas a la distancia del $\mathrm{X}^{2}$ como el método exacto de Fisher o la corrección de Yates (Sáenz de Buruaga 1991: 69). En base a la intensidad de estas rupturas, disponemos de un reconocimiento jerarquizado de las interrelaciones internas. Siguiendo a C. Guillamón (Vila 1986: 75) ${ }^{4}$, los límites de significación empleados como unidad básica de medición de la intensidad de la ruptura son los habituales en el uso del $\mathrm{X}^{2}(\mathrm{p}<0.05, \mathrm{p}<0.01, \mathrm{p}<0.001)$.

De esta forma obtenemos la secuencia estructural (Laplace 1972 y 1974) de cada una de las variables morfotécnicas de los instrumentos para un mismo proceso de trabajo concreto. Se obtienen así las dinámicas forma-función y se jerarquizan los instrumentos de cada proceso de trabajo en base a su distancia con otros. Los resultados de la secuencia estructural pueden ser presentados gráficamente, en donde el símbolo "/" nos indica la presencia de una ruptura, y el número de repeticiones del mismo, es indicador de progresivos incrementos de intensidad de la misma hasta llegar al grado máximo representado por "///". La simbología " ", por el contrario, indica continuidad, es decir, ausencia de ruptura. Los numerales nos indican el número

4 Los resultados presentados aquí se han obtenido mediante la aplicación informática diseñada por J. Estévez a partir de los algoritmos de G. Laplace. de casos presente para cada variable en el análisis concreto (sobre la aplicación estadística concreta: Vila 1986). Como ejemplo, el resultado para el análisis de la variable tipo de filo para los instrumentos no retocados que han procesado fauna(genérico) ${ }^{5}$ es:

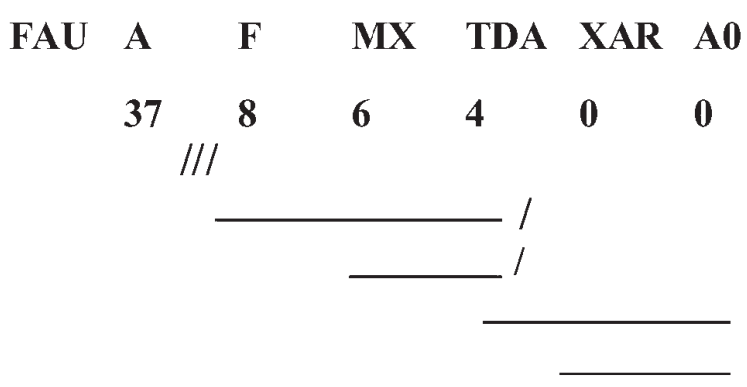

Fig. 2. Ejemplo de Secuencia Estructural.

Los tipos de filo presentes en este tipo de instrumento son: ángulo $(A)^{6}$, fractura $(F)$, ausencia de una tendencia definitoria (MX), Tendencia ángulo (TDA), charnela (XAR) y ángulo cero (A0). En el gráfico, podemos apreciar, a efectos de interpretación, básicamente tres niveles de jerarquización significativa:

- la presencia destacada (con una ruptura respecto al resto marcada como muy significativa: "///") del uso de ángulos.

- la presencia de una ruptura significativa en aquellas categorías que no tienen ningún caso (charnela y ángulo cero) y la restantes, ruptura que estaría provocada por la contraposición cuantitativa, producto del aporte nulo de estos dos últimos valores. La estabilidad entre los restantes valores (fragmentos, mixtos y tendencia al uso de ángulos) nos permite apuntar que el uso de ángulos es, ampliamente, el rasgo morfotécnico seleccionado para la producción de estos instrumentos.

La Dinámica Estructural, por el contrario nos permite, nuevamente a través del test $\mathrm{X}^{2}$, la comparación entre diferentes unidades comprobando cuál es la variación existente entre ellas y si esa variación tiene una significación matemática (Sáenz de Buruaga 1991: 73). Es decir: si existe una variabilidad causal dentro de un conjunto determinado en relación, por

\footnotetext{
5 Los procesos de trabajo que implican fauna(genérico) son: piel, carne, hueso y fauna.

6 Filo natural.
} 


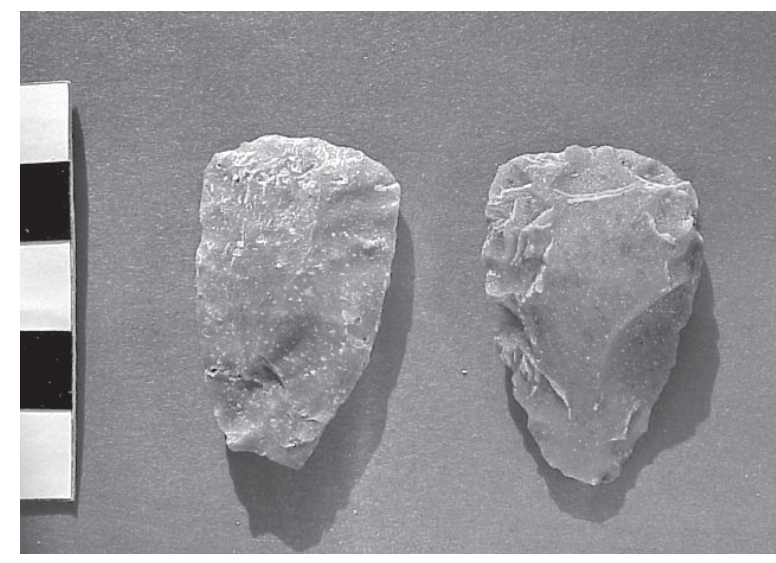

Fig. 3. Instrumental lítico de Túnel VII.

ejemplo a las cinemáticas de trabajo, para las dinámicas morfológicas identificadas como relevantes (Briz 2004) (1991: 73) los niveles de significación para determinar las variaciones inter-conjunto son las siguientes: -homogeneidad ( $p>$.010), -homogeneidad con reserva $(.005<p<.010)$, -heterogeneidad significativa $(.001<\mathrm{p}<.005)$, -heterogeneidad muy significativa $(.0001<\mathrm{p}<.001)$, -heterogeneidad altamente significativa $(\mathrm{p}<.0001)$ (Sáenz de Buruaga 1991: 73).

El resultado gráfico de la aplicación del análisis para evaluar la interrelación entre el tipo de filo empleado (Tendencia de uso de filo natural (TDA), Filo natural (A), Fractura (F), Sin tendencia definida (MX), punta (A0) y charnela (XAR)) y la materia trabajada: carne (CAR), fauna (FAU), piel (PIEL), madera (MAD) y hueso (HU) es el siguiente:

En este caso, la única ruptura interesante a nivel de la distribución de los casos (que se representa con el símbolo "/") se encuentra en la correlación entre el uso de filos naturales para el procesado del hueso (por contraposición al resto de materias trabajadas); sin embargo no se trata de una diferencia lo suficientemente significativa como para considerarla

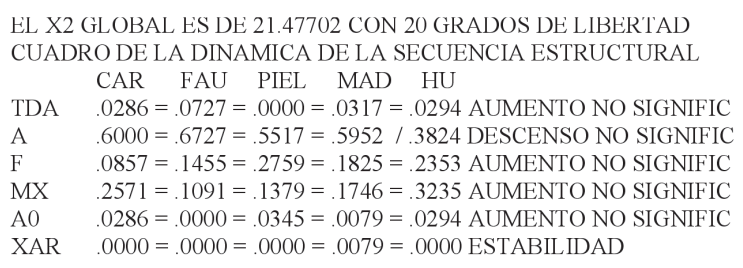

Fig. 4. Ejemplo de la Dinámica de la Secuencia Estructural. relevante. Por lo tanto no existe una correlación entre tipo de filo y materia trabajada.

\section{TÚNEL VII, RESULTADOS}

El conjunto lítico de Túnel VII está conformado por un total de 22.154 ítems líticos de aporte antrópico (Briz 2004). De ese total, 22.043 han sido identificados como resultado de estrategias de producción (Terradas 1996) y fueron analizados a nivel morfotécnico y sólo un 1'88\% presenta formatización secundaria. El análisis funcional fue realizado por Ignacio Clemente quien puso en relieve las capacidades del método en contextos cazadoresrecolectores-pescadores de Tierra del Fuego (Clemente (1997). Los resultados de ese análisis permitieron determinar que sobre un total de 20.814 artefactos, el 96'34\% no presentaban rastros de uso (94'93\%) o exhibían alteraciones postdepositacionales (1'41\%). El 3'66 \% ( $n=762)$ restante son instrumentos de trabajo microscópicamente contrastados, con un total de 939 filos activos. Emplearemos este último conjunto para explorar las dinámicas forma-función.

Como ya hemos indicado anteriormente, el análisis de las posibles pautas de correlación entre el uso y las morfologías se inicia en una primera caracterización del conjunto a nivel de correlaciones generales sin entrar a analizar, aún, las dinámicas internas de las variables morfologicas de los filos activos. Conseguimos así, una primera orientación de interrelaciones forma-función que se verá complementada por el segundo análisis estadístico. Es importante destacar que lo que buscamos son pautas y no estados: consideraremos como relevantes cambios significativos en las tendencias de los conjuntos y no sólo cambios globales en los conjuntos. Empezaremos este primer análisis por la correlación entre el uso de filos retocados y el uso: de los 939 filos activos, 265 son retocados $\left(28^{\prime} 22 \%\right)^{7}$.

Nuestra hipótesis de trabajo en este caso específico es la existencia de una correlación entre la mayor inversión de trabajo (presencia de formatización secundaria en los filos activos) y el proceso de uso (cinemática de trabajo, dureza de la materia trabajada y materia trabajada; esta última, articulada

7 Los datos y representaciones gráficas de los resultados expuestos en los párrafos siguientes son fácilmente accesibles, vía internet (Briz 2004: 345 y ss): http://www. tesisenred.net/TDX-1215104-163111 


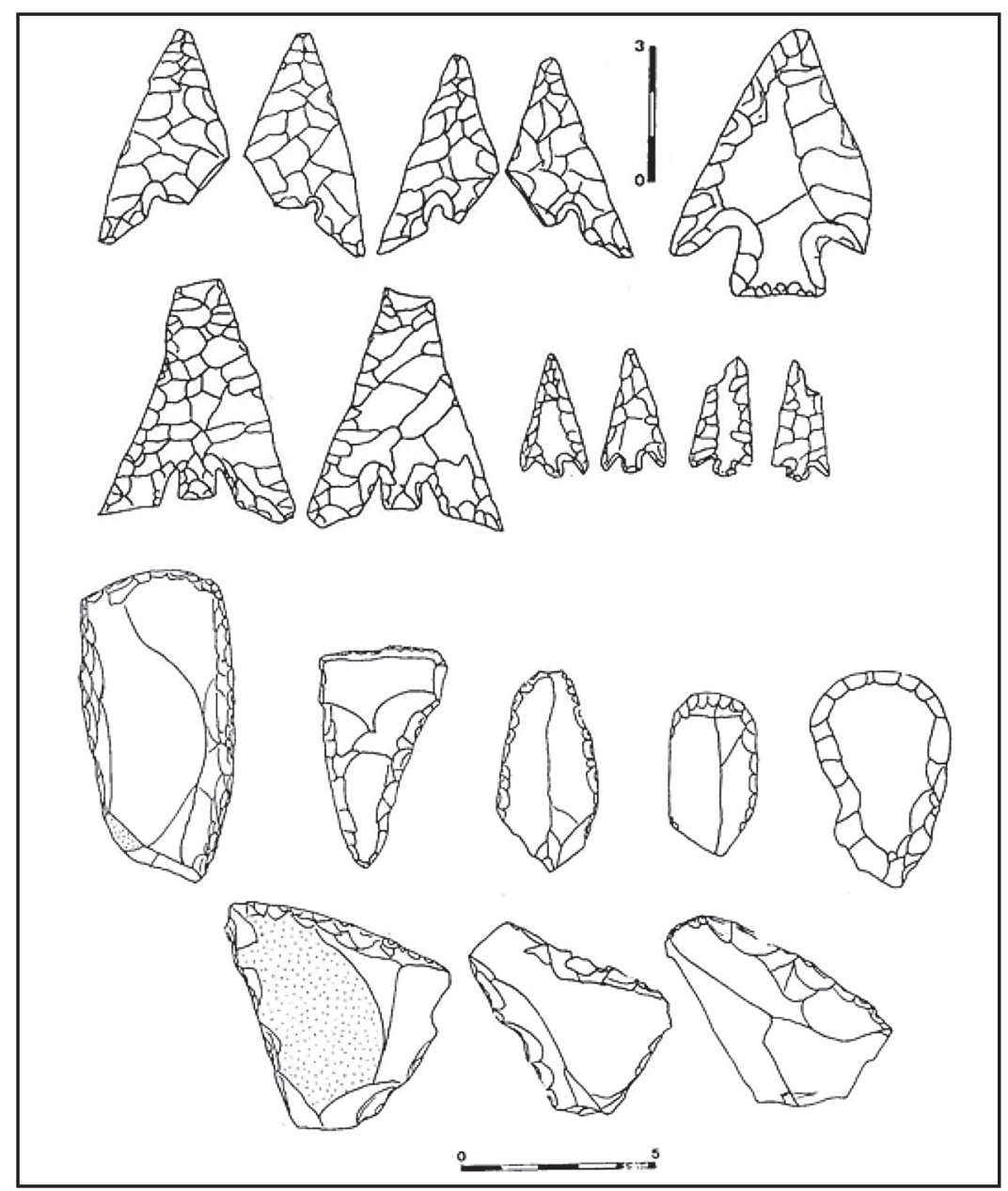

Fig. 5. Artefactos e instrumentos de Túnel VII.

en una gradación en base a su dureza, que complementa la anterior).

Si articulamos el conjunto en base a las las cinemáticas de trabajo, se constató un incremento de filos retocados en relación a las cinemáticas transversales, del $21 \%$ al $41 \%$, con una disminución de los no retocados de $79 \%$ a $51 \%$. Si, por el contrario, las correlaciones son en base a la dureza de la materia trabajada, apreciamos una interrelación, también, en relación a las materias duras: 62'5\% retocados y 37 '5\% no retocados. Relación que se invierte en una progresión más que destacada si se trata de materias blandas: $11^{\prime} 4 \%$ retocados y $88^{\prime} 6 \%$ no retocados.

Identificadas estas dos dinámicas, se pudo emplear la tercera articulación del conjunto (en relación a la materia trabajada) como elemento que nos permita discriminar entre las correlaciones existentes. Los resultados no mostraron, en este caso, una gradación progresiva relacional entre la formatización secundaria y la especificidad de la materia trabajada. Si, además incrementamos la escala de nuestro análisis, para analizar la variabilidad del retoque en función de la cinemática de trabajo de la única materia trabajada que, por su volumen de casos, puede ser analizada independientemente y a nivel interno, (el trabajo de madera) se destacó una clara tendencia de incremento de la formatización secundaria en el paso de las cinemáticas longitudinales a las transversales (del 18\% al 40\%, con una disminución de las piezas no retocadas del $82 \%$ al $60 \%$ ). Apuntando, consecuentemente, a la existencia de una correlación entre presencia de retoque y cinemática de trabajo como la más importante. 


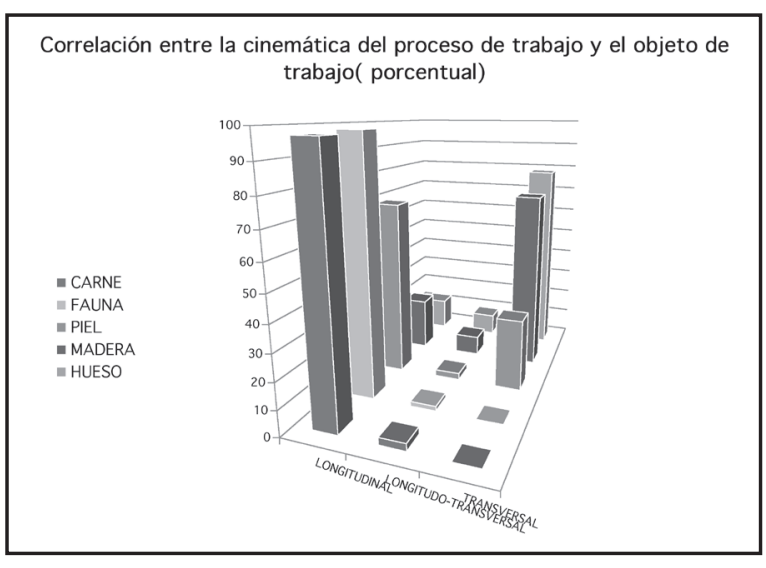

Fig. 6. Correlación entre cinemática del proceso de trabajo y la materia trabajada (porcentual).

Para contrastar esta primera visión, se organizaron los mismos conjuntos desde nuevas perspectivas. Al articular el conjunto en base a la correlación entre cinemática de trabajo y dureza, se mostró que en las materias blandas o blandas-medias, hay preeminencia de los trabajos longitudinales, con una mínima presencia de longitudo-transversales y casi inexistentes para los transversales. Para las materias con dureza media, media-dura y dura, la dinámica se invierte, pese a que la diferencia entre la cinemática dominante (transversal) y la secundaria es muy leve. Si contrastamos la materia trabajada con la cinemática surge, finalmente, una visión explicativa de la inversión de trabajo en formatización secundaria, donde existe una clara adscripción de la materia trabajada a un tipo determinado de cinemática. Hay un neto predominio de actividades de corte sobre carne y materiales faunísticos en contraposición con el trabajo sobre madera y hueso donde predominan las cinemáticas transversales.

No existe una única causa de correlación forma-función para la formatización secundaria. Los análisis más detallados de cada una de las materias trabajadas (Briz 2004: 349 y ss.) terminaron de perfilar estos resultados: independientemente de su dureza, determinadas materias (tales como carne, fauna(genérico), piel y madera) poseen una correlación entre la presencia de retoque y la cinemática de trabajo. Los instrumentos retocados que habían trabajado fauna o carne habían sido empleados en base a dinámicas longitudinales. El trabajo de piel, sin embargo, mostraba una asociación entre la presencia de retoque y la cinemática transversal.
Dinámica que se repitió en el caso específico de la madera (pese a que en este caso, no se llega a invertirse la ratio entre instrumentos retocados y no retocados).

Existe, sin embargo, una segunda dinámica en relación a la producción de instrumentos con retoque, y es en relación a los trabajos sobre hueso donde la importancia del retoque es mayor: 66'6\% para los trabajos longitudinales y longitudo-transversales, y 68 '5\% para cinemática transversal. Ante las variaciones de las cinemáticas de trabajo, la estabilidad en la importancia de la formatización secundaria es absoluta. La incidencia del retoque está estrechamente vinculado, entonces, a las condiciones concretas (dureza) de esta materia trabajada (Briz 2004: 356).

Una vez evaluada la correlación entre retoque y función, pasamos a emplear la secuencia estructural y la dinámica estructural para identificar cuáles son las morfologías relevantes en correlación a los procesos de trabajo desarrollados, independientemente de la presencia o ausencia de formatización secundaria. Puesto que previamente y hemos revisado y expuesto el método empleado, brindaremos directamente los resultados a nivel de caracterización de las dinámicas ${ }^{8}$.

El sistema de representación empleado intenta conservar la coherencia respecto a la lógica de los utilizados anteriormente, empleando la siguiente simbología:

- "/" = equivalencia alterna de ambas variables (la posición indica tan sólo preeminencia, no ruptura); -"()" = ausencia de dinámica característica forma-función;

- “***)" =tendencia existente en un rango jerarquico determinado".

Los resultados de secuencia estructural en base a la cinemática, la dureza del objeto de trabajo y el mismo objeto de trabajo se observan en las siguientes tablas. En el caso del trabajo específico sobre madera, el elevado número de casos nos permite, además, poder analizarlo por separado, consiguiendo una mayor resolución. Los resultados que se ofrecen son los rasgos morfológicos característicos para cada proceso de trabajo, articulado para evidenciar los diferentes aspectos del mismo:

8 Para una revisión más en detalle del proceso e interpretación de los resultados de la dinámica estructural consultar Briz 2004: 366-374).

9 En donde ** son rasgos morfológicos. 
Tabla 2. Secuencias estructurales resultantes. En base a la cinemática de trabajo.

\begin{tabular}{|c|c|c|c|}
\hline \multirow{7}{*}{ 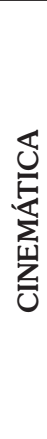 } & \multicolumn{3}{|c|}{ Instrumentos sin formatización secundaria } \\
\hline & Longitudinal & Longitudo-Transversal & Transversal \\
\hline & aSrect/sin & $\mathrm{a}()()$ & $\mathrm{a}(\mathrm{f} / \mathrm{mx})$ Arect/sin \\
\hline & $\begin{array}{l}\text { La dinámica característica es el } \\
\text { uso de ángulos (a) Simples (S), } \\
\text { rectilíneos (rect) o sinuosos (sin). }\end{array}$ & $\begin{array}{l}\text { Empleo de ángulos sin ningún otro } \\
\text { rasgo asociable a la función. }\end{array}$ & $\begin{array}{l}\text { Uso de ángulos (con una segunda } \\
\text { tendencia conformada por el uso } \\
\text { de fracturas o filos mixtos) de modo } \\
\text { abrupto rectilíneos o sinuosos. }\end{array}$ \\
\hline & \multicolumn{3}{|c|}{ Instrumentos con formatización secundaria } \\
\hline & $\mathrm{rSm} / \mathrm{ppd} /$ bifrect & $\mathrm{r}()()()()$ & $\mathrm{rSm} / \mathrm{ppdrect} / \mathrm{cx}$ \\
\hline & $\begin{array}{l}\text { Retoque de modo simple marginal o } \\
\text { profundo directo o bifacial rectilíneo }\end{array}$ & Presencia de retoque & $\begin{array}{l}\text { Retoque Simple marginal o } \\
\text { profundo direct con delineación } \\
\text { rectilínea o convexa }\end{array}$ \\
\hline
\end{tabular}

Tabla 3. Secuencias estructurales resultantes. En base a la dureza de la materia trabajada.

\begin{tabular}{|c|c|c|c|c|c|}
\hline \multirow{7}{*}{ 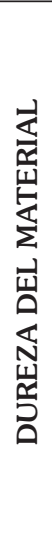 } & \multicolumn{5}{|c|}{ Instrumentos sin formatización secundaria: } \\
\hline & Blandas & Blandas-Medias & Medias & Medias-Duras & Duras \\
\hline & aSrect/sin & $\mathrm{a}($ )rect/sin & $\mathrm{a}() \sin / \mathrm{rect}$ & ()Arect/sin & () $\mathrm{A}(\mathrm{MX})()$ \\
\hline & $\begin{array}{l}\text { Ángulo simple } \\
\text { rctilíneo o sinuoso }\end{array}$ & $\begin{array}{l}\text { Angulo, sin modo } \\
\text { determinado, } \\
\text { rectilíneo o sinuoso }\end{array}$ & $\begin{array}{c}\text { Ángulo, sin modo } \\
\text { determinado, } \\
\text { sinuoso o rectilíneo }\end{array}$ & $\begin{array}{l}\text { Filos de modo abrupto } \\
\text { rectilíneo o sinuosos }\end{array}$ & $\begin{array}{l}\text { Filos abruptos o de } \\
\text { tendencia no definida }\end{array}$ \\
\hline & \multicolumn{5}{|c|}{ Instrumentos con formatización secundaria } \\
\hline & $\mathrm{rP} / \mathrm{S}($ )bif(d)cx/rect & $\mathrm{rS}() \mathrm{d}()$ & $\mathrm{rS}()$ drect & $\mathrm{r}()() \mathrm{d}()$ & $\mathrm{rS}($ )drect/cx \\
\hline & $\begin{array}{l}\text { Retoques planos o } \\
\text { simples sin tendencia } \\
\text { definida en la } \\
\text { amplitud bifaciales } \\
\text { o, en segundo nivel, } \\
\text { directos, convexos } \\
\text { o rectilíneos }\end{array}$ & $\begin{array}{l}\text { Retoques simples } \\
\text { directos }\end{array}$ & $\begin{array}{l}\text { Retoques simples } \\
\text { directos rectilíneos }\end{array}$ & Retoques directos & $\begin{array}{l}\text { Retoques simples } \\
\text { directos rectilíneos } \\
\text { o convexos }\end{array}$ \\
\hline
\end{tabular}

Tabla 4. Secuencias estructurales resultantes. En base a la materia trabajada.

\begin{tabular}{|c|c|c|c|c|c|}
\hline \multirow{6}{*}{ 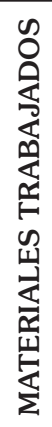 } & Carne & Fauna & Piel & Madera & Hueso \\
\hline & $\mathrm{a}($ )rect/sin/mix & aSrect/sin & $\mathrm{a} / \mathrm{f}($ )rect/sin/cx & $\mathrm{a}() \sin / \mathrm{rect}$ & ()()() \\
\hline & $\begin{array}{l}\text { Ángulo rectilíneo o } \\
\text { sinuoso o mixto }\end{array}$ & $\begin{array}{l}\text { Ángulo simple } \\
\text { rectilíneo o sinuoso }\end{array}$ & $\begin{array}{l}\text { Ángulo o fractura } \\
\text { sin Modo definido, } \\
\text { rectilíneo o } \\
\text { sinuoso convexo }\end{array}$ & $\begin{array}{l}\text { Ángulo sinuoso } \\
\text { o rectilíneo }\end{array}$ & $\begin{array}{l}\text { No se identifican } \\
\text { tendencias. }\end{array}$ \\
\hline & \multicolumn{5}{|c|}{ Instrumentos con formatización secundaria } \\
\hline & $\mathrm{r}()() \mathrm{bif} / \mathrm{drect} / \mathrm{cx}$ & $\mathrm{r}()()()()$ & $\mathrm{rS} / \mathrm{P}() \mathrm{drect} / \mathrm{cx}$ & rSmdrect & $\mathrm{rSpp} / \mathrm{mdrect} / \mathrm{cx}$ \\
\hline & $\begin{array}{l}\text { Retoque sin modo } \\
\text { definido bifacial o } \\
\text { directo rectilíneo } \\
\text { o convexo }\end{array}$ & Uso de retoque & $\begin{array}{l}\text { Retoque simple } \\
\text { o plano, directo, } \\
\text { rectilíneo o convexo }\end{array}$ & $\begin{array}{l}\text { Retoque simple } \\
\text { marginal directo } \\
\text { rectilíneo }\end{array}$ & $\begin{array}{l}\text { Retoque simple, } \\
\text { muy profundo o } \\
\text { marginal, directo } \\
\text { rectilíneo o convexo }\end{array}$ \\
\hline
\end{tabular}

Tabla 5. Secuencia estructural específica de los trabajos de madera.

\begin{tabular}{|c|c|c|c|}
\hline \multirow{7}{*}{ 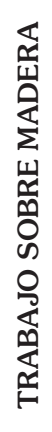 } & \multicolumn{3}{|c|}{ Instrumentos sin formatización secundaria } \\
\hline & Longitudinal & Longitudo-Transversal & Transversal \\
\hline & aSrect/sin & $\mathrm{a}()()$ & $\mathrm{a}(\mathrm{f} / \mathrm{mx})$ Arect/sin \\
\hline & $\begin{array}{l}\text { La dinámica característica es el } \\
\text { uso de ángulos (a) Simples (S), } \\
\text { rectilíneos (rect) o sinuosos (sin). }\end{array}$ & $\begin{array}{l}\text { Empleo de ángulos sin ningún otro } \\
\text { rasgo asociable a la función. }\end{array}$ & $\begin{array}{l}\text { Uso de ángulos (con una segunda } \\
\text { tendencia conformada por el uso } \\
\text { de fracturas o filos mixtos) de modo } \\
\text { abrupto rectilíneos o sinuosos. }\end{array}$ \\
\hline & \multicolumn{3}{|c|}{ Instrumentos con formatización secundaria } \\
\hline & $\mathrm{rSm} / \mathrm{ppd} /$ bifrect & $\mathrm{r}()()()()$ & $\mathrm{rSm} / \mathrm{ppdrect} / \mathrm{cx}$ \\
\hline & $\begin{array}{l}\text { Retoque de modo simple marginal o } \\
\text { profundo directo o bifacial rectilíneo }\end{array}$ & Presencia de retoque & $\begin{array}{l}\text { Retoque Simple marginal o } \\
\text { profundo direct con delineación } \\
\text { rectilínea o convexa }\end{array}$ \\
\hline
\end{tabular}


IVAN BRIZ

Tabla 6. Dinámicas forma-función características para materias trabajadas no óseas.

\begin{tabular}{|c|c|c|c|}
\hline \multirow{7}{*}{ 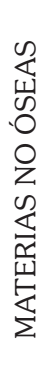 } & \multicolumn{3}{|c|}{ Instrumentos sin formatización secundaria } \\
\hline & Longitudinal & Longitudo-Transversal & Transversal \\
\hline & aSrect/sin & $\mathrm{a}()()$ & $\mathrm{a}(\mathrm{f} / \mathrm{mx})$ Arect/sin \\
\hline & Ángulo simple, rectilíneo o sinuoso & Uso de ángulo & $\begin{array}{c}\text { Ángulo (en un segundo nivel fractura } \\
\text { o sin tendencia identificada) de modo } \\
\text { abrupto, rectilínieo o sinuoso. }\end{array}$ \\
\hline & \multicolumn{3}{|c|}{ Instrumentos con formatización secundaria } \\
\hline & $\mathrm{rSm} / \mathrm{ppd} /$ bifrect & $\mathrm{r}()()()()$ & $\mathrm{rSm} / \mathrm{ppdrect} / \mathrm{cx}$ \\
\hline & $\begin{array}{l}\text { Retoque simple, marginal o muy } \\
\text { profundo, directo o bifacial, rectilíneo. }\end{array}$ & Uso de retoque. & $\begin{array}{l}\text { Retoque simple marginal o muy } \\
\text { profundo, directo, recto o convexo. }\end{array}$ \\
\hline
\end{tabular}

Si bien mediante el uso articulado de la secuencia y la dinámica estructural hemos localizado estas morfologías como relevantes a nivel forma-función, necesitamos poder seleccionar las formas de organización de los resultados (en base a la materia, a la cinemática, etc...) que consideremos más efectivas para la interpretación económica.

El análisis realizado previamente sobre los rasgos primarios (formatización secundaria vs. ausencia de formatización secundaria) se incorpora aquí, al ser aquellos resultados los elementos que, complementado la información que acabamos de revisar, nos permiten articular el reconocimiento final de las dinámicas de nuestros conjuntos. Y es empleado para ambas categorías: formatizadas y no. La interrelación es dialéctica: el incremento de una implica el descenso de la otra; y desde el momento en que existe una causa identificada para ese incremento, esa es la misma causa explicativa para el comportamiento de los opuestos y su descenso.

Hemos visto previamente cómo todos los instrumentos que no han trabajado hueso se articulan en base a la cinemática de trabajo; mientras que los trabajos sobre hueso se articulan en base a la dureza específica del objeto de trabajo concreto. En base a esto, los resultados para las materias trabajadas no óseas, a nivel de las secuencias estructurales, son (Briz 2004: 366 y ss):

Tabla 7. Secuencia estructural específica del trabajo sobre hueso (en base a su dureza).

\begin{tabular}{cc}
\hline TRABAJO SOBRE HUESO (en base a dureza) \\
\hline Sin formatización & Con formatización \\
{$[() \mathrm{A}(\mathrm{MX})(\mathrm{)})$} & {$[\mathrm{rS}() \mathrm{drect} / \mathrm{cx}]$} \\
$\begin{array}{c}\text { modo abrupto o bien } \\
\text { sin tendencia }\end{array}$ & $\begin{array}{c}\text { retoque simple, directo, } \\
\text { rectilíneo o convexo }\end{array}$ \\
\hline
\end{tabular}

En lo que respecta al trabajo sobre hueso, articularemos su análisis en base al criterio de dureza: la tendencia es el uso de cualquier tipo de filo de modo abrupto, o bien sin tendencia, para aquellas piezas sin formatización secundaria, y el uso de retoque simple, directo, rectilíneo o convexo para los piezas retocadas.

$\mathrm{Si}$, además, incrementamos la escala del análisis para reconocer los rasgos característicos los resultados del trabajo sobre hueso en base a su especificidad los rasgos son la ausencia de cualquier pauta para los instrumentos sin formatización secundaria; y el retoque simple, muy profundo o marginal, directo, rectilíneo o convexo, para los instrumentos con formatización secundaria. Consecuentemente, tendremos que emplear el criterio de dureza anteriormente mencionado para los no retocados.

Consecuentemente, en el caso de los instrumentos no formatizados para hueso, emplearemos el criterio de dureza, mientras que los formatizados podemos emplear un instrumento analítico de mayor resolución mediante el uso de la secuencia estructural en base al objeto de trabajo. Éstos serán los rasgos morfológicos que caracterizarán estas dinámicas forma-función.

Sin embargo, nos queda pendiente la caracterización de un elemento destacado de cualquier conjunto lítico: los instrumentos que han sido identificados como para la obtención de recursos animales. En

Tabla 8. Secuencia estructural específica del trabajo sobre hueso.

\begin{tabular}{cc}
\hline \multicolumn{2}{c}{ TRABAJO SOBRE HUESO (específico) } \\
\hline Sin formatización & Con formatización \\
{$[(()())]$,} & {$[\mathrm{rSpp} / \mathrm{mdrect} / \mathrm{cx}]$,} \\
Sin pauta & $\begin{array}{c}\text { retoque simple, muy } \\
\text { profundo o marginal, } \\
\text { directo, rectilíneo o convexo }\end{array}$ \\
\hline
\end{tabular}


el caso de Túnel VII, este conjunto está conformado por un total de 23 piezas, todas ellas con formatización secundaria, y que los trabajos de I. Clemente (1997) clasificaron como "Punta", "Daga", "Puñal" o "Cuchillo". La determinación de su uso posee la dificultad de la ausencia de evidencias claras, dado el poco tiempo de contacto con la materia trabajada. Diversas líneas de trabajo apuntan que la presencia de una fractura distal (o bien el ápice fragmentado de una pieza) son elemento definidores de su uso, pues se trata de fracturas de impacto (Finlayson y Mithen 1997; Nelson 1997). Para conocer los trabajos que se están realizando en estos momentos en Tierra del Fuego: Álvarez e.p.). En este caso, es el instrumento en su globalidad el que ha de ser evaluado como partícipe en el proceso de trabajo. Prácticamente la totalidad presentan retoque plano muy profundo (o profundo) bifacial, con presencia destacada de ángulo 0 o fractura abrupta para el tramo distal de la pieza. Las delineaciones de los filos izquierdo y derecho (que habíamos dejado de lado en los instrumentos anteriores), nos indican una clara preeminencia de la convergencia. Los rasgos característicos para los filos laterales será, así pues: [rPpp/pbifrect cvg] acompañados bien de ángulo 0 o fractura abrupta distal.

Lo que hemos logrado hasta este punto del trabajo es identificar las dinámicas forma-función para cada uno los instrumentos empleados en los distintos procesos. Para cada proceso de trabajo han sido producidas y empleadas unas morfologías específicas. El conjunto de las morfologías significativas empleadas en cada uno de estos procesos, así como la estructuración de la variabilidad interna de este mismo conjunto, es lo que convenimos en denominar dinámica. La variabilidad interna de las morfologías de los intrumentos concretos empleados en cada proceso de trabajo, se articulará en una diversidad más o menos próxima a los comportamientos característicos de esa dinámica forma-función (Briz 2004; Briz et al. 2002 y 2005), estableciéndose una jerarquía de relaciones forma-función más o menos próxima al comportamiento reconocido como característico para ese proceso de trabajo. De esta forma, podemos reconocer, midiéndola, la diversidad existente dentro de unidades socialmente significativas (Lumbreras 1984a y b), bien en base a su delimitación como proceso de trabajo (por ejemplo, el trabajo de corte de hueso), bien como momento de desarrollo de actividades humanas (un

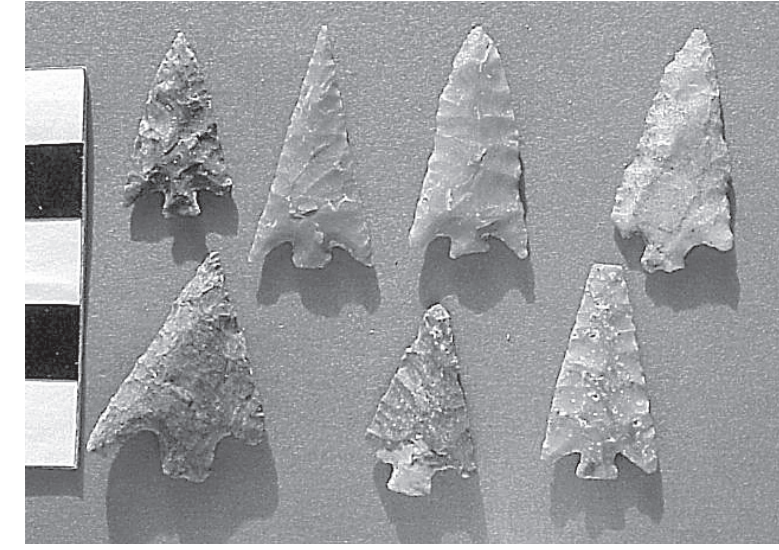

Fig. 7. Artefactos/Instrumentos para la caza.

sitio arqueológico, un episodio de ocupación como los que aquí nos interesan, etc...).

Clasificaremos los instrumentos de producción identificados en una triple categorización jerárquica: consideraremos Instrumento (I) todo aquél del cual únicamente podemos identificar su participación en un proceso productivo, sin poder aportar ninguna otra información funcional o morfotécnica.

Categorizaremos como Instrumento de Dinámica Característica (IDC) aquellos instrumentos que cumplen con los rasgos morfotécnicos identificados para la dinámica significativa, seguido del proceso productivo implementado, o en su defecto, la materia trabajada (IDC(PP/OT)). En esta categoría, consideraremos la existencia de diferentes niveles jerárquicos, a nivel analítico-interpretativo, en aquellas dinámicas que no sean las significativas $\left(\mathrm{IDC}_{2}(\mathrm{PP})\right.$ $\mathrm{OT}), \mathrm{IDC}_{3}$, etc...), según el caso presente en cada una de las variables caracterizadoras del proceso de trabajo (cinemática o materia trabajada): $\mathrm{IDC}_{2}$ (procesado longitudinal madera) o $\mathrm{IDC}_{3}$ (procesado madera). Los restantes Instrumentos de Producción no asimilables a las dinámicas predominantes, serán identificados como Instrumentos (I(PP/OT)). El resto de elementos del conjunto han sido identificados, a efectos de este análisis, en diferentes categorías: núcleos (N), residuos (RSD) y aportes líticos (AL). La primera, referida a la masa lítica a partir de la que se obtienen soportes básicos; entendida como materia prima del proceso productivo de artefactos líticos por talla. La segunda (RSD) reconoce aquellos ítems que presentan rasgos identificativos de haber sido modificados morfológicamente por el trabajo, pese a que no presentan indicios de ser un 
instrumento ni sus morfologías son relacionables dentro del reconocimiento de aquellos artefactos morfológicamente iguales a un instrumento pero no consumidos (rechazos) ${ }^{10}$. Ahora bien, en este subconjunto (residuos), existen una dinámica interna: existen, por un lado, aquellos residuos de reducida volumetría que no presentan ningún rasgo asimilable a los rechazos al mismo tiempo que no poseen ningún indicio de tipo funcional. Pero, al mismo tiempo, existen aquellos residuos que, sin poseer ningún rasgo morfológico para ser asimilados a los rechazos, sí poseen indicios de uso. La aparición de este tipo de ítems es relacionable con la participación de los artefactos líticos como instrumentos de trabajo y su consecuente amortización. Si este residuo secundario no posee evidencias de talla intencional (talón, punto de impacto, etc...), podemos considerarlo como residuo de la propia participación instrumental del artefacto, considerándolo Residuo de Instrumento (RSDI). Ahora bien, si presentan evidencias de producción intencional por percusión o presión, nos encontramos ante una adición de trabajo a un instrumento ya empleado, con el objetivo de prolongar su vida útil. Se tratará, entonces, de Residuos de Instrumentos Revalorizados (RSDIR). Finalmente, los Aportes Líticos (AL) serán aquellos elementos que, pese a no presentar ningún indicio de trabajo invertido en la modificación de su morfología o su uso instrumental, su presencia en nuestros contextos estratigráficos tan sólo puede ser interpretada en base a un orígen antrópico.

Caracterizados en base a esta sistemática cada uno de los instrumentos, podremos caracterizar y analizar los momentos históricos concretos de Túnel VII.

\section{Revisemos algunos ejemplos}

El instrumento número 2487, perteneciente al episodio de ocupación E, posee un uso contrastado, en su filo izquierdo, sobre madera con cinemática longitudinal. El análisis morfológico del filo activo nos ofrece los siguientes rasgos: [rSmbirect div-aScc cvg]. La dinámica significativa de relación formafunción identificada para este proceso de trabajo

10 Pese a que no emplearemos esta categoría analítica en este trabajo, para profundizar en la definición de los artefactos morfológicamente iguales a un instrumento pero no consumidos: Briz et al. 2002 y 2005. es la siguiente: [rSm/ppd/bifrect]. Es decir: retoque simple, marginal o muy profundo, directo o bifacial, rectilíneo. Consecuentemente, el instrumento 2487, en su filo izquierdo, posee los rasgos característicos de la dinámica forma-función para el trabajo longitudinal de madera, puediendo ser catalogado com carcaterístico: IDC(MLO); en donde IDC = instrumento de dinámica característica, $(\mathrm{MLO})=$ trabajo de madera longitudinal.

El instrumento inventariado como 23887, con trabajo longitudinal sobre hueso fresco en el filo derecho, posee las siguientes características: [aSsin div-fAcc div]. La ausencia en este caso de algunos de los rasgos característicos de la dinámica del trabajo longitudinal sobre hueso [()A(MX)()] nos llevan a clasificarlo en un segundo nivel jerárquico respecto a la dinámica característica: IDC2(HFLO) (instrumento de dinámica característica de segundo nivel para el trabajo longitudinal de hueso fresco).

\section{CARACTERIZANDO UN MOMENTO EN MOVIMIENTO: LAS DINÁMICAS FORMA-FUNCIÓN DE LAS OCUPACIONES DE TÚNEL VII}

Traslademos nuestros resultados a los contextos históricos específicos de las diferentes ocupaciones de Túnel VII. Es decir: reconocer la estructura de los conjuntos instrumentales en cada uno de los episodios de ocupación del sitio, en base a las clasificación que acabamos de generar. En este trabajo nos hemos concentrado única y exclusivamente en aquellas dinámicas donde se reconoce plenamente el proceso de trabajo (materia trabajada y cinemática de trabajo) además de la dinámica genérica "instrumento".

La presentación gráfica de qué trabajos se desarrollaron en las ocupaciones seleccionadas (algunos de los episodioss, por su baja cantidad de instrumentos, no han sido tratados) y cuál es la diversidad (jerarquizada) del instrumental empleado, nos ofrece una clara visión de las dinámicas producción-consumo de cada una de los momentos de actividad y vida de Túnel VII.

Las codificaciones empleadas, que antes ya hemos observado, son: el primer acrónimo nos informa de la identificación y jerarquización del instrumento. El segundo, (entre paréntesis), hace referencia al proceso de trabajo concreto (Objeto de trabajo más cinemática). 


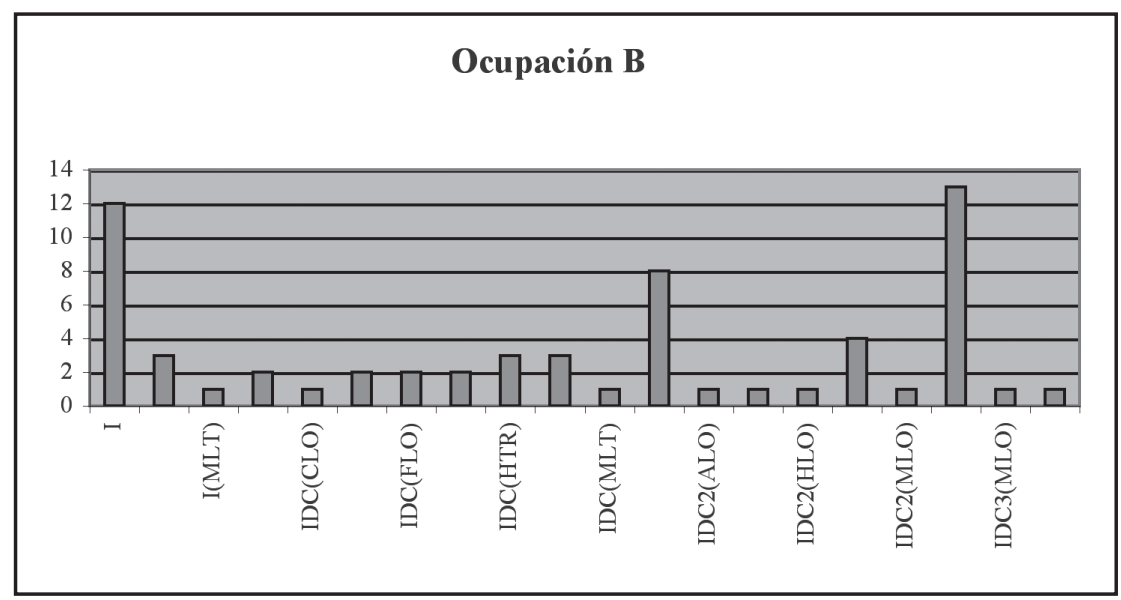

Fig. 8. Ejemplo de gráfica descriptiva (absoluta) para el episodio de ocupación B. Referencias: I: Instrumento.

IDC: Instrumento de Dinámica Característica. IDC2, 3....: Instrumento de Dinámica Característica Jerarquizada (donde el numeral subíndice nos indica su distancia respecto a la dinámica característica típica). RSDI: Residuo de Instrumento. RSDIR: Residuo de Instrumento Revalorizado. L: Lítico. C: Carne. F: Fauna. P: Piel. M: Madera. H: Hueso. LO: Longitudinal. LT: Longitudo-Transversal. TR: Transversal.

Los episodiso caracterizados:

breves descripciones

\section{El Episodio B}

La ocupación B de Túnel VII se caracteriza por la destacada preeminencia de los trabajos sobre madera con instrumental característico de primer y segundo grado, siempre con cinemáticas transversales. En el caso del segundo, fijémonos cómo su importancia es superior, incluso, a la categoría genérica de "instrumento". Una preeminencia muy similar a la de los trabajos transversales (también) sobre hueso de morfologías próximas a la tipicidad (primer y tercer grado, con una curiosa reducción en el segundo grado). Los resultados de la secuencia estructural para el mismo (Briz 2004: CD-ROM), marcaban como significativa la segmentación de estos dos grupos respecto al resto de trabajo en hueso. Un tercer elemento de este grupo destacado son los trabajos longitudinales sobre fauna. El resto de las dinámicas existentes no presentan, en este rápido análisis, elementos a destacar.

\section{El Episodio C}

Como en el caso anterior, la preeminencia de trabajos sobre madera y hueso se mostró más que destacable (caracterizada, a nivel de la secuencia estructural, con una interrupción significativa). Pero en el caso del primero de los dos procesos de trabajo, la dinámica del instrumental está mucho más alejada de la dinámica significativa que el episodio anterior (A): los subconjuntos más significativos se ubicaron en los grados 3 y 2 de representatividad. Al mismo tiempo, y para el trabajo transversal sobre hueso, la dinámica significativa estaba claramente representada y acompañada de un subconjunto de grado jerárquico 3. Al observar la dinámica de la secuencia estructural, se pudo observar cómo el conjunto de instrumentos "genéricos" pasó a ocupar una posición preeminente esperable. A destacar, también, la aparición del instrumental para la obtención de animales.

\section{El Episodio D}

La variabilidad de los procesos de producción, en este caso, fue marcadamente superior a los anteriores. Como grupo muy diferenciado se mostró el instrumento "genérico", concentrándose nuestro análisis en el segundo subconjunto. Los trabajos transversales de madera y hueso continuron siendo preeminentes, donde el uso de instrumento característico para hueso es importante. En el caso de la madera transversal, el instrumental de segundo grado jerárquico se mostró como el más destacado. El procesado de carne y fauna se desarrolla con instrumental muy lejano de la dinámica característica (indicando poca especialización morfotécnica respecto al comportamiento general). 


\section{El Episodio E}

En el caso del episodio E, la variabilidad de las dinámicas se mostró aún superior al anterior. El instrumental clasificado como "genérico" y el procesado transversal de madera en grados jerárquicos de nivel 2 y 3 conformaron el primer grupo significativo. Las dinámicas para el procesado de la madera son similares a las del episodio anterior (estaríamos hablando de organizaciones del trabajo muy similares), aunque a nivel de los trabajos desarrollados globalmente, el trabajo en hueso desciende, de fomra absoluta, en importancia. En el segundo subconjunto significativo, apareció, de nuevo, una presencia destacada del trabajo sobre madera (en esta ocasión, empleando dinámicas características para el procesado transversal) y de jerarquía de grado 3 para el longitudinal. El procesado de fauna se alejaba de la dinámica significativa (estamos hablando, consecuentemente, de una mayor variabilidad de la relación forma-función), mientras que el trabajo sobre hueso se caracterizaba por un elevado grado de especificidad respecto a la dinámica característica o de segunda jerarquía. Se trata, entonces, de una obtención de artefactos muy específica y cuidadosa.

Finalmente, destacar cómo en este segundo subconjunto, existe una preeminencia en número de casos del procesado de fauna por delante del trabajo específico del hueso, acompañada de la presencia de dos instrumetnos para la obtención de fauna.

\section{El Episodio F}

A nivel de los procesos productivos implementados, $\mathrm{F}$ es el conjunto con mayor nivel de representatividad (por el número de casos) y, al mismo tiempo, variabilidad. Curiosamente, no hemos podido identificar ninguna pauta secuencial a nivel de su estructura. La más importante interrupción muy significativa que se nos mostró, implicaba al instrumental "genérico", claramente diferenciado del resto. El experimento de repetir el análisis en esta categoría (como evaluación que estuviese actuando como atractor extraño de la totalidad del conjunto) no nos ofreció una lectura diferenciada. De nuevo, la importancia de los trabajos sobre madera es preeminente, con una más que marcada importancia de los instrumenos de dinámica significativa. A nivel de dinámicas secundarias del episodio, vemos aparecer los trabajos sobre piel que se desarrolla con dinámicas característica o muy próxima a la característica. Los trabajos sobre hueso se mantienen dentro de dinámicas similares a las anteriores. Y, pese a que no implica ninguna relevancia a nivel de la dinámica de la secuencia estructural, dado el bajo número de efectivos, destacar la aparición de instrumental para el procesado de vegetales, que no madera, en base a dinámicas próximas a la especificidad significativa.

\section{El Episodio G}

Este episodio no nos ofreció ningún cambio significativo respecto a las anteriores pautas. Los trabajos tanto de madera como de hueso (ambos en cinemáticas transversales), continúan con las mismas dinámicas observadas en los casos anteriores. En todo caso, apuntar que el instrumental de dinámica significativa para el procesado de carne y fauna, alcanzaba una preeminencia interesante respecto a la producción y uso de morfologías más diversas, pero acompañado de una importante presencia de instrumentos para el procesado de fauna muy alejado de la dinámica significativa: en grado de jerarquía 4.

\section{El Episodio $\mathrm{H}$}

En estos últimos episodios de ocupación, la significación de la dinámica estructural es prácticamente nula. Si no tenemos en cuenta la significación relevante (por otro lado, esperable) del comportamiento diferencial del instrumental "genérico", no se apreció ninguna interrupción significativa en la secuencia estructural. Tan sólo comentar, pese a su nulo nivel de significación, la dinámica significativa del procesado de fauna, que se situó a un mismo nivel que el trabajo sobre madera y los trabajos sobre piel. Destacar, eso sí, un interesante indicador de los niveles de aprovechamiento del instrumental: aparecen, por primera vez, residuos de revalorización del instrumental empleado para el trabajo de piel.

\section{El Episodio J}

Pese a su bajo número de componentes, este episodio sí nos ofrece dinámicas estructurales significativas. El procesado de fauna mediante instrumentos que participan de la dinámica significativa, o muy próximos (grado jerárquico 2) es destacado, 


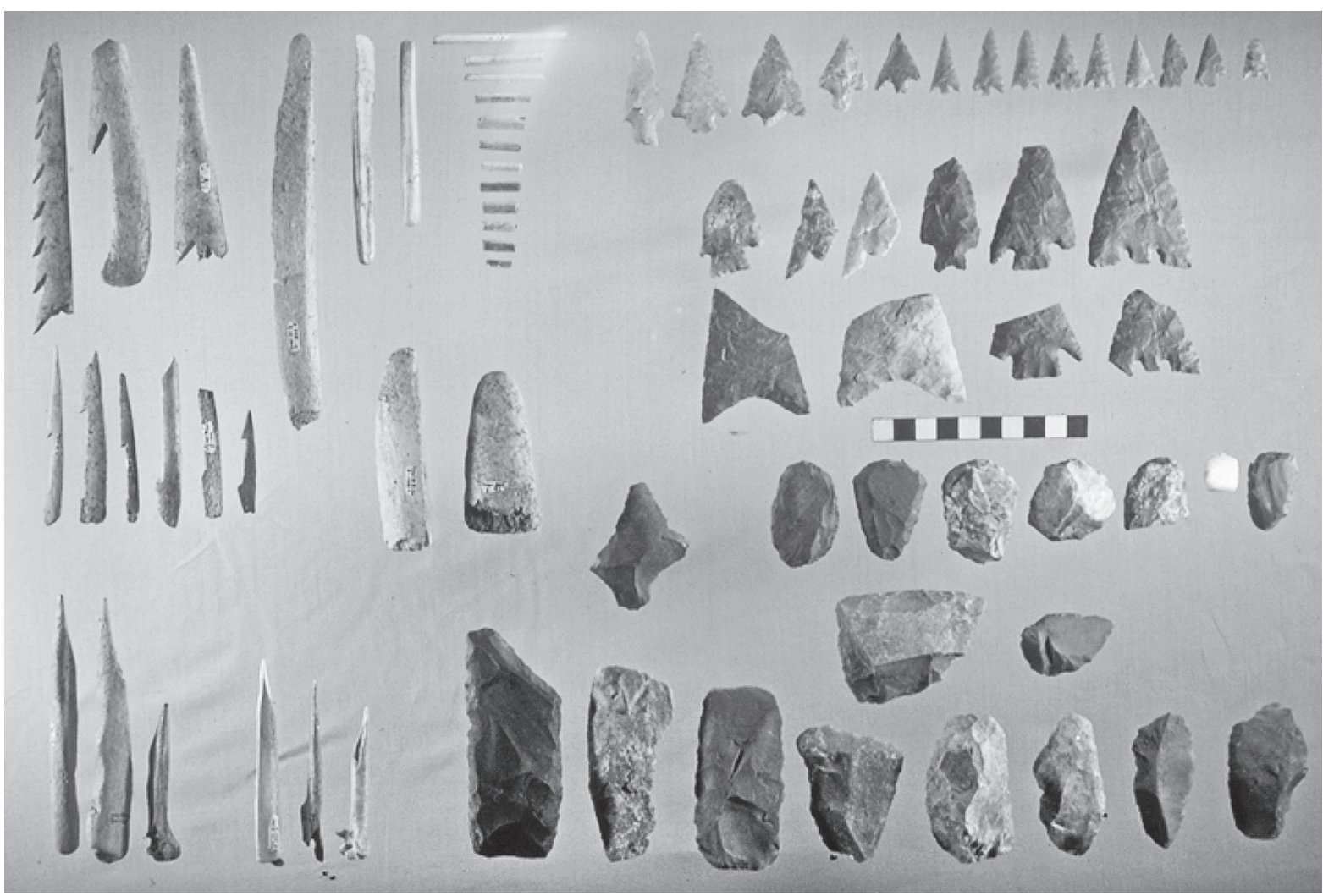

Fig. 9. Conjunto representativo de artefactos e instrumentos líticos y óseos de Túnel VII.

junto con el procesado de madera transversal en instrumentos de dinámica de grado secundario. El resto de las dinámicas identificadas nos permiten únicamente constatar la presencia de producciones: madera, fauna, carne y hueso; con ausencia de trabajos de procesado de piel.

\section{CONSIDERACIONES FINALES}

A través de estas páginas ha intentado proponer un método para la clasificación analítica del instrumental lítico que nos permita revelar la relación forma-función que había sido enmascarada por el empleo de las tipologías tradicionales. Mediante este método proponemos, como punto de partida, el contexto de uso (Álvarez 2003) de los artefactos líticos como eje para establecer asociaciones significativas entre las variables morfotécnicas y descubrir las decisiones socio-económicas que subyacen a los procesos de gestión y consumo de recursos. Al abandonar la preconcepción tipológica de los artefactos líticos e iniciar nuestra aproximación en base a un planteamiento analítico del análisis morfológico, que permita reconocer su complejidad, abrimos la vía para poder contrastar la presencia del uso como elemento esencial en el reconocimiento de la producción y aprovechamiento de las morfologías líticas.

La aplicación a los materiales de Túnel VII ha permitido corroborar en primera instancia que la inversión de trabajo en la manufactura de instrumentos está vinculada en forma significativa con la dureza de los materiales trabajados y con la cinemática. Hay un destacado predominio de la utilización de filos retocados para el desarrollo de acciones transversales (raspado, alisado) sobre materiales duros (madera y hueso) y de filos naturales para el corte de sustancias blandas (piel, carne). Los dos únicos materiales en que esta relación es menos contrastante son: la piel, que experimenta un leve crecimiento en lo que respecta a las actividades transversales, y la madera en la que las actividades de corte son un poco más frecuentes que en el resto de los materiales duros. Estos resultados muestran que el retoque, que ha sido utilizado por muchas tipologías tradicionales 
como criterio para la definición de tipos, puede ser analizado como una estrategia para aumentar la efectividad del trabajo sobre materiales específicos. Pero lo más importante es trazar en estas páginas que nuestro métodos, aún en fase de desarrollo, son capaces de mostrarnos toda la dinámica económica que produjo, y subyace, en materiales supuestamente estáticos e inertes. Pretendemos reconocer (y medir) la variabilidad de las relaciones forma-función en un conjunto de instrumentos líticos, para poder clasificarlos, posteriormente y de forma analítica, en base a su actividad económica. Los resultados presentados permiten caracterizar instrumentos $y$ episodios de ocupación en base a los rasgos dinámicos de los conjuntos que los conforman. Rasgos dinámicos que surgen en base a la presunción de la naturaleza económica de estos instrumentos. Si el movimiento es la base de nuestra metodología de análisis y reconocimiento de la industria lítica, la variabilidad, el cambio, es la esencia de la misma. Cambio y tiempo son inseparables, son el núcleo del devenir histórico que es el objeto de nuestra ciencia.

En estas páginas se ha pretendido presentar las posibilidades de la propuesta que hemos aplicado a Túnel VII. No es una propuesta definitiva, cerrada. Nuestra prioridad ha sido intentar exponer de forma lo más sencilla posible las bases sobre las que se asienta su posterior desarrollo, intentando seguir paso a paso este último con la intención de ofrecer a nuestros colegas nuevas vías de trabajo a las que aproximarse, analizar y, sin duda, intentar mejorar. Coherente con sus propios orígenes, la propuesta está abierta y necesita una constante revisión, crítica y desarrollo. Trabajos que se estan desarrollando en estos momentos nos permitirán presentar resultados más completos y afinados de esta misma metodología aplicada a contextos líticos fueguinos.

Finalmente, agradecer sinceramente al comité editor de Magallania y las personas evaluadoras de este trabajo su ayuda para hacer más comprensibles estas páginas.

\section{BIBLIOGRAFÍA}

ADAMS, W. 1988, Archaeological Classification: theory versus practice. Antiquity 64(234): 40-56.

ÁlVAREZ, M. 2003. Organización Tecnológica en el Canal Beagle. El caso de Túnel I (Tierra del Fuego, Argentina). Tesis doctoral. Universidad de Buenos Aires, Buenos Aires.
ÁLVAREZ, M. e.p. Puntas de arma del extremo sur de Patagonia: algunas consideraciones sobre diseño y contexto de uso. En: Armas prehispánicas: múltiples enfoques para su estudio en Sudamérica. Bozzuto, D y Martínez, J. (eds.). Editorial Fundación Azara, Buenos Aires.

ÁLVAREZ, M. y BRIZ, I. 2006. Organización tecnológica en el proceso de poblamiento del extremo Sur de Sudamérica. HABITUS. GOIANIA. Revista do Instituto Goiano de Pré-História e Antropologia 4(2):771-795.

ANDREFSKY, W. 1998. Lithics. Macroscopic approaches to analysis, Cambridge Manuals in Archaeology, Cambridge University Press, Cambridge.

BATE, L.F. 1982. Relación general entre teoría y método en arqueología. Teorías, Métodos y Técnicas en Arqueología. Reimpresiones de Antropología Americana núm. especial: 3-50.

BRIZ, I. 2002. Producción y Consumo. En: Análisis Funcional. Su aplicación al estudio de sociedades prehistóricas. Clemente, I., Risch, R. y Gibaja, J. (eds.). British Archaeological Reports. International Series, 1073, Archeopress, Oxford, Pp.: 43-51.

BRIZ, I. 2004. Dinàmiques econòmiques de producció-consum en el registre lític caçador-recol-lector de l'extrem sud americà. La societat Yàmana. Publicación electrónica del Servei de Publicacions de la Universitat Autònoma de Barcelona, Barcelona. http://www.tesisenred.net/ TDX-1215104-163111

BRIZ, I. 2007. Piedras, dinámicas, producciones y consumos: propuesta desde la Dialéctica para el análisis de conjuntos líticos. KREI 9: 27-46.

BRIZ, I. 2010. Etnoarcheologia: che cosa, come, verso dove? Quaderni di Thule. Rivista d'Americanistica IX: 549-559.

BRIZ, I., CLEMENTE, I., PIJOAN, J., TERRADAS, X. Y VILA, A. 2002. Contextos etnorqueològics i l'estudi de Conjunts Lítics. Cota Zero. Revista de Ciència $i$ Arqueologia 17: 12-20.

BRIZ, I., CLEMENTE, I., PIJOAN, J., TERRADAS, X. y VILA, A. 2005. Stone tools in etnoarchaeological contexts: theoretical-methodological inferences. En: Lithic Toolkits in Ethnoarchaeological Contexts. Actts of the XIVth UISPP Congress. Liège, Belgium. 2001. Terradas, X. (ed.). British Archaeological Reports, International Series, S1370, Archeopress, Oxford, Pp.: 1-7.

BRIZ, I., ÁlVAREZ, M., SPIKINS, P. y NEEDHAM, A. 2009. "Durable residues": Addressing the use of microwear, a case study from March Hill. Internet Archaeology 26. http://intarch.ac.uk/journal/issue26/godino_index.html 
CALVO, M. 1999. Reflexiones en torno al concepto de útil, forma, función y su relación con los análisis funcionales. Pyrenae 30: 17-38.

CALVO, M. 2002. Útiles líticos prehistóricos. Forma, función y uso. Ariel Prehistoria, Ariel, Barcelona.

CARBONELL, E. y RODRÍGUEZ, X.P. 2002. El Sistema Lògic Analític: origen, desenvolupament i perspectives de futur. Cota Zero. Revista de Ciència i Arqueologia 17:106-116.

CLARKE, D.L. 1984 [1968]. Arqueología Analítica. Ediciones Bellaterra, Barcelona, $2^{\mathrm{a}}$.

CLEMENTE, I. 1997. Los Instrumentos líticos de Túnel VII: una Aproximación etnoarqueológica. Treballs d'Etnoarqueologia, 2, UAB-CSIC, Madrid.

CLEMENTE, I., MANSUR, E., TERRADAS, X. y VILA, A. 1996. Al césar lo que es del césar... o los "instrumentos" líticos como instrumentos de trabajo. En: Arqueología. Sólo Patagonia. Ponencias de las Segundas Jornadas de Arqueología de la Patagonia. Gómez, J. (ed.). Centro Nacional Patagónico-CONICET, Río Gallegos, Pp.: 319-331.

DE GORTARI, E. 1965. Introducción a la Lógica Dialéctica, FCE, México, 3ㅁ.

DE GORTARI, E. 1970. El Método Dialéctico, Ed. Grijalbo, México.

DEBERT, J. y SHERRIFF, B. L. 2007. Raspadita: a new lithic tool from the Isthmus of Rivas, Nicaragua. Journal of Archaeological Science 34(11): 1889-1901.

ESTÉVEZ, J. y VILA, A. (coords.) 1996. Encuentros en los conchales fueguinos, Treballs d'Etnoarqueologia, 1, CSIC-UAB, Madrid.

ESTÉVEZ, J. y VILA, A. 1998. Tierra del Fuego, lugar de encuentros. Revista de Arqueología Americana 15: 187-219.

ESTÉVEZ, J. y VILA, A. 2000. Estratigrafías en contexto. KREI 5: 29-61.

ESTÉVEZ, J. y VILA, A. 2006. Variability in the lithic and faunal record through 10 reoccupations of a XIX century Yamana Hut. Journal of Archaeological Science 25(4): 408-423.

FINLAYSON, B. y MITHEN, S. 1997. The Microwear and Morphology of Microliths from Gleann Mor. en: Projectile Technology. Knecht, H. (ed.), Plenum press, New York, Pp.: 107-129.

JUEL JENSEN, H. 1988. Functional Analyis of Prehistoric Flint Tools by High-Power Microscopy: A Review of West European Research. Journal World of Prehistory 2(1): 53-88.

HARDY, B., BOLUS, M. y CONARD, N. 2008. Hammer or crescent wrench? Stone-tool form and function in the aurignacian of southwest Germany. Journal of Human Evolution 54(5): 648-662.
HISCOCK, P. 2007. Looking the other way. A materialist/ technological approach to classifying tools and implements, cores and retouched flakes. En: Tools versus Cores? Alternative approaches to Stone Tool Analysis, McPherron, S. (ed.). Cambridge Scholars Publishing, Cambridge, Pp.: 198-222.

INGOLD, T. 1997. Eight themes in the Anthropology of technology. Techology as skilled practice. Social Analysis 41(1): 106-138.

INGOLD, T. 2001. Ecologia della cultura. Meltemi editore, Roma KNECHT, H. 1988. Upper Paleolithic Burins. Type, Form and Function. British Archaeological Reports. International Series, 434, Archeopress, Oxford.

KOROBKOVA, G.F. 1983. Development of the Productive forces and of working tools as a prerequisite of the evolutions of new types of economy. Journal of Central Asia VI(1): 73-80.

LANDINI, C., BONOMO, M., LEIPUS, M. y MARTÍNEZ, G. 2000. Forma y función de los instrumentos líticos del sitio Paso Otero 3 (Pdo. De Necochea, pcia. de Buenos Aires, Argentina): un estudio comparativo. Espacio,Tiempo y Forma, Serie I, Prehistoria y Arqueología (13): 161-187.

LAPLACE, G. 1956. Typologies statistiques et évolution des complexes à lames et lamelles. Bulletin de la Société Préhistorique Française LIII(6): 271-290.

LAPLACE, G. 1964. Essai de Typologie Systématique, Universitá degli studi di Ferrara, Ferrara.

LAPLACE, G. 1966. Recherches sur l'Origine et l'Évolution des Complexes Leptolithiques, Mélanges d'Archéologie et d'Histoire. Suppl. 4, École Française de Rome, Paris.

LAPLACE, G. 1972. La Typologie analytique et structurale: Base rationelle d'études des industries lithiques et osseusses. Sep. Banques de donneés Archéologiques. Colloques Nationaux du CNRS, CNRS, Marseille.

LAPLACE, G. 1974. De la Dynamique de l'Analyse structurale ou la typologie analytique. Sep. Rivista di Scienze Preistoriche XXIX(1), Firenze.

LAPLACE, G. 1975. Distance du Khi 2 et algorithmes de Classification Hierarchique. Dialektikê. Cahiers de Typologie Analytique 1975: 22-37.

LAPLACE, G. 1980. Le lien comme mesure de l'information dans un tableau de contingence. Dialektikê. Cahiers de Typologie Analytique, 1980: 1-15.

LAPLACE, G. 1981. Algorithme de segmentation de la matrice d'Homogeneité. Dialektikê. Cahiers de Typologie Analytique 1981: 15-28.

LAPLACE, G. y LIVACHE, M. 1975. Precissions sur la demarche de l'Analyse Structurale. Dialektikê. Cahiers de Typologie Analytique 1975: 8-21. 
LONGO, L. y SKAKUN, N. (eds.) 2005. The roots of Use-Wear Analysis: selected papers of S. A. Semenov. Published on the occasion of the Congress "Prehistoric Technology" 40 years later: functional studies and the Russian Legacy. Memorie del Museo Civico di Storia Naturale di Verona Sezione Scienze dell'Uomo, 7, Verona.

LUMBRERAS, L.G. 1984a La Unidad Arqueológica Socialmente Significativa (I). Gaceta Arqueológica Andina 1(10): 3

LUMBRERAS, L.G. 1984b. La Unidad Arqueológica Socialmente Significativa (II). Gaceta Arqueológica Andina 1(11): 3.

MANSUR, M. E. 1987. El análisis funcional de artefactos líticos. Cuadernos Serie Técnica 1, Instituto Nacional de Antropología, Buenos Aires.

MANSUR, M. E. y LASA, A. 2005, Diversidad artefactual vs. especialización funcional. Análisis del IV componente de Túnel I (Tierra del Fuego, Argentina). Magallania 33(2): 69-91.

MARX, K. 1992 [1867]. El Capital. Crítica de la Economía Política. 3 vol., Fondo de Cultura Económica, México, $2^{\mathrm{a}}$ ed. $23^{\mathrm{a}}$ reimp.

MERINO, J.M. 1994. Tipología Lítica. MUNIBE (Antropologia-Arkeologia). Supl. 9, Aranzadi Zientzi Elkantea, Donostia, 3.

NELSON, M.C. 1997. Projectile points. Form, function, and design. En: Projectile Technology. Knecht, H. (ed.). Plenum press, New York, Pp.: 371-384.

ODELL, G.H. 1981. The Morphological express at function junction: searching for meaning in Lithic tool types. Journal of Anthropological Research (37): 319-342.

ORQUERA, L. 1996. Túnel VII: la estratigrafía. En: Encuentros en los conchales fueguinos. Estévez, J. y Vila, A. (coords.). Treballs d'etnoarqueologia, 1, UAB-CSIC, Barcelona, Pp.: 83-103.

ORQUERA, L. y PIANA, E. 1996a. Túnel VII: la excavación. En: Encuentros en los conchales fueguinos. Estévez, J. y Vila, A. (coords.). Treballs d'etnoarqueologia, 1, UAB-CSIC, Barcelona, Pp.: 47-81.

ORQUERA, L. y PIANA, E. 1996b. Túnel VII: la cronología. En: Encuentros en los conchales fueguinos. Estévez, J. y Vila, A. (coords.). Treballs d'etnoarqueologia, 1, UAB-CSIC, Barcelona, Pp.: 105-111.

ORQUERA, L. y PIANA, E. 1999. Arqueología de la Región del canal Beagle (Tierra del Fuego, República Argentina). Sociedad Argentina de Antropología, Buenos Aires.

ORQUERA, L. y PIANA, E. 2000. Composición de conchales de la Costa del Canal Beagle (Tierra del Fuego, República Argentina) -Primera Parte-. Relaciones de la Sociedad Argentina de Antropología XXV: 249-274.
PAWLIK, A. F. 2009. Is the functional approach helpful to overcome the typology dilemma of lithic archaeology in southeast asia?. IPPA Bulletin 29: 6-14

PFAFFEMBERGER, B. 1992. Social anthropology of technology. Annual Review of Anthropology 21: 491-516.

PIANA, E. L., VILA, A., ORQUERA, L.A. y ESTÉVEZ, J. 1992. Chronicles of Ona-ashaga: Archaeology in the Beagle Channel. Antiquity 252: 771-783.

PIÉ, J. y VILA, A. 1992. Relaciones entre objetivos y métodos en el estudio de la Indústria Lítica. En: Tecnología y Cadenas Operativas Líticas. Reunión internacional, 15-18 enero de 1991, Mora, R., Terradas, X., Parpal, A. y Plana, F. (eds.). Treballs d'Arqueologia, UAB, Barcelona, Pp.: 271-278.

PIQUÉ, R. 1999. Producción y uso del combustible vegetal: una evaluación arqueológica. Treballs d'Etnoarqueologia, 3, CSIC-UAB, Madrid.

RISCH, R. 2002. Análisis funcional y Producción social: relación entre método arqueológico y teoría económica. En: Análisis Funcional. Su aplicación al estudio de sociedades prehistóricas. Clemente, I., Risch, R. y Gibaja, J. (eds.). British Arcaheological Reports. International Series, 1073, Archeopress, Oxford, Pp.: 19-29.

SÁENZ DE BURUAGA, A. 1991. El Paleolítico Superior de la Cueva de Gatzarria Zuberoa, Pais Vasco. Veleia. Anejos, 6, Instituto de Ciencias de la AntigüedadServicio Editorial de la Universidad del Pais Vasco, Gasteiz.

SEMENOV, S. A. 1971. Fonctionologie du Paléolithique. VII Congrès International des Sciences Préhistoriques et Protohistoriques (Belgrade, 1971). Les rapports et les communications de la Délégation des archéologues de l'URSS, Moscú, Separata.

SEMENOV, S. A. 1981[1957]. Tecnología prehistórica. Estudio de las Herramientas y Objetos antiguos a través de las Huellas de Uso. Akal, Madrid.

TERRADAS, X. 1996. La Gestió dels Recursos Minerals entre les Comunitats Caçadores-Recolectores. Vers una Representació de les Estratègies de Proveïment de Matèries Primeres. Tesis Doctoral. Universitat Autònoma de Barcelona, Barcelona.

VILA, A. 1977. Analyse fonctionnelle et Analyse morphotechnique. Dialektikê. Cahiers de Typologie analytique 1977: 54-58

VILA, A. (ed.) 1986. Introducció a l'estudi de les Eines Lítiques Prehistòriques. Universitat Autònoma de Barcelona, Barcelona.

VILA, A. 2004. Proyectos etnoarqueológicos en Tierra del Fuego. Bienes Culturales 3: 193-200. 
VILA A. 1985. El "Cingle Vermell": Assentament de CaçadorsRecol.lectors del Xè Mil.leni B.P. Generalitat de Catalunya, Barcelona.

VILA, A. y CLEMENTE, I 2000. Reflexiones en torno al Congreso-Homenaje a S. Semenov. Revista AtlánticoMediterránea de Prehistoria y Arqueología Social 3: 345-354.

VILA, A. y ESTÉVEZ, J. 2000. El Paleolítico que nos enseñaro. Actas do $3^{\circ}$ Congresso de Arqueologia Peninsular. V.I. Arqueologia Peninsular. Historia, Teoria e Prática, ADECAP, Porto, Pp.: 345-365.

VILA, A., y ESTÉVEZ, J. 2001. Calibrando el método: arqueología en Tierra del Fuego. Astigi Vetus 1: 63-72.

VILA, A. y PIANA, E. 1993. Arguments per a una etnoarqueologia. Revista d'Etnologia de Catalunya 3: 151-154.

VILA, A., PIANA E., ESTÉVEZ, J. y ORQUERA, L. 1996a. Encuentros en los conchales fueguinos. En: Encuentros en los conchales fueguinos. Estévez, J. y Vila, A. (coords.), Treballs d'etnoarqueologia, 1, UAB-CSIC, Barcelona, Pp.: 5-16.
VILA, A., TERRADAS, X., CLEMENTE, I. y MANSUR, E. 1996b. La larga marcha: de roca a instrumento. en: Encuentros en los conchales fueguinos. Estévez, J. y Vila, A., (coords.). Treballs d'Etnoarqueologia, 1, UABCSIC, Barcelona, Pp.: 261-273.

VILA, A., MAMELI, L., TERRADAS, X., ESTÉVEZ, J., MORENO, F., VERDÚN, E., BRIZ, I., ZURRO, D., CLEMENTE, I. y BARCELÓ, J. A. 2007. Investigaciones etnoarqueológicas en Tierra del Fuego (1986-2006): reflexiones para la Arqueología Prehistórica Europea. Trabajos de Prehistoria 64 (2): 37-54.

ZURRO, D. 1996. El análisis de fitolitos y su papel en el estudio del consumo de recursos vegetales en a prehistoria: bases para una metodológica materialista. Trabajos de Prehistoria 63(2): 35-54.

ZURRO, D. 2010. Ni carne ni pescado (consumo de recursos vegetales en la Prehistoria). Análisis de la variabilidad de conjuntos fitolitológicos en contextos cazadoresrecolectores. Tesis doctoral. Universitat Autònoma de Barcelona, Barcelona. 
